\title{
Sun Earth Connection Coronal and Heliospheric Investigation (SECCHI)
}

\author{
R.A. Howard · J.D. Moses • A. Vourlidas · J.S. Newmark • D.G. Socker • \\ S.P. Plunkett • C.M. Korendyke · J.W. Cook • A. Hurley • J.M. Davila • \\ W.T. Thompson • O.C. St Cyr · E. Mentzell $\cdot$ K. Mehalick $\cdot$ J.R. Lemen • \\ J.P. Wuelser · D.W. Duncan - T.D. Tarbell • C.J. Wolfson - A. Moore · R.A. Harrison • \\ N.R. Waltham • J. Lang • C.J. Davis • C.J. Eyles • H. Mapson-Menard • \\ G.M. Simnett · J.P. Halain · J.M. Defise · E. Mazy • P. Rochus · R. Mercier • \\ M.F. Ravet · F. Delmotte · F. Auchere · J.P. Delaboudiniere · V. Bothmer • \\ W. Deutsch • D. Wang • N. Rich • S. Cooper • V. Stephens • G. Maahs • R. Baugh • \\ D. McMullin - T. Carter
}

Received: 30 November 2006 / Accepted: 12 March 2008 / Published online: 16 May 2008 (C) Springer Science+Business Media B.V. 2008

R.A. Howard $(\varangle) \cdot$ J.D. Moses $\cdot$ A. Vourlidas $\cdot$ J.S. Newmark · D.G. Socker $\cdot$ S.P. Plunkett ·
C.M. Korendyke $\cdot$ J.W. Cook · A. Hurley
E.O. Hulburt Center for Space Research, Naval Research Laboratory, Washington, DC 20375, USA
e-mail: russ.howard@ nrl.navy.mil

J.M. Davila · W.T. Thompson · O.C. St Cyr · E. Mentzell · K. Mehalick NASA Goddard Space Flight Center, Greenbelt, MD 20742, USA

J.R. Lemen · J.P. Wuelser · D.W. Duncan · T.D. Tarbell · C.J. Wolfson · A. Moore Lockheed Martin Solar and Astrophysics Lab., Palo Alto, CA 94304, USA

R.A. Harrison · N.R. Waltham · J. Lang · C.J. Davis

Space Science and Technology Dept., Rutherford Appleton Laboratory, Chilton, Didcot, Oxfordshire OX11 OQX, UK

C.J. Eyles · H. Mapson-Menard · G.M. Simnett

Astrophysics and Space Research Group, University of Birmingham, Edgbaston, Birmingham B15 2TT, UK

J.P. Halain · J.M. Defise · E. Mazy · P. Rochus

Centre Spatiale de Liège, Université de Liège, Avenue du Pré-Aily, 4031 Angleur, Belgium

R. Mercier · M.F. Ravet · F. Delmotte

Laboratoire Charles-Fabry de l'Institut d'Optique (IOTA), Campus Polytechnique, RD 128-91227,

Palaiseau Cedex, France

F. Auchere - J.P. Delaboudiniere

Institut d'Astrophysique Spatiale, Centre universitaire d'Orsay, Bât 120-121, 91405 Orsay Cedex,

France

V. Bothmer

Max-Planck-Institute for Solar System Research, Institute for Astrophysics, University of Göttingen, 37077 Göttingen, Germany 
Abstract The Sun Earth Connection Coronal and Heliospheric Investigation (SECCHI) is a five telescope package, which has been developed for the Solar Terrestrial Relation Observatory (STEREO) mission by the Naval Research Laboratory (USA), the Lockheed Solar and Astrophysics Laboratory (USA), the Goddard Space Flight Center (USA), the University of Birmingham (UK), the Rutherford Appleton Laboratory (UK), the Max Planck Institute for Solar System Research (Germany), the Centre Spatiale de Leige (Belgium), the Institut d'Optique (France) and the Institut d'Astrophysique Spatiale (France). SECCHI comprises five telescopes, which together image the solar corona from the solar disk to beyond 1 AU. These telescopes are: an extreme ultraviolet imager (EUVI: 1-1.7 $\mathrm{R}_{\odot}$ ), two traditional Lyot coronagraphs (COR1: 1.5-4 $\mathrm{R}_{\odot}$ and COR2: $2.5-15 \mathrm{R}_{\odot}$ ) and two new designs of heliospheric imagers (HI-1: 15-84 $\mathrm{R}_{\odot}$ and HI-2: 66-318 $\mathrm{R}_{\odot}$ ). All the instruments use $2048 \times 2048$ pixel CCD arrays in a backside-in mode. The EUVI backside surface has been specially processed for EUV sensitivity, while the others have an anti-reflection coating applied. A multi-tasking operating system, running on a PowerPC CPU, receives commands from the spacecraft, controls the instrument operations, acquires the images and compresses them for downlink through the main science channel (at compression factors typically up to $20 \times$ ) and also through a low bandwidth channel to be used for space weather forecasting (at compression factors up to $200 \times$ ). An image compression factor of about $10 \times$ enable the collection of images at the rate of about one every 2-3 minutes. Identical instruments, except for different sizes of occulters, are included on the STEREO-A and STEREO-B spacecraft.

Keywords Solar corona - Lyot coronagraph · XUV heliograph · Heliospheric imager · Coronal loops $\cdot$ Coronal mass ejections $\cdot$ Stereo $\cdot$ Heliosphere

\title{
1 Introduction
}

The Sun-Earth-Connection Coronal and Heliospheric Investigation (SECCHI; see Howard et al. 2000) for the NASA Solar Terrestrial Relations Observatory (STEREO) mission is a suite of optical telescopes that will, for the first time, observe the entire inner heliosphere from the solar surface out to the vicinity of Earth. By combining this very large field of view with the radio and in-situ measurements from STEREO as well as from other spaceand earth-based assets, we expect to answer some important questions of the physics of Coronal Mass Ejections (CMEs). The instrument acronym is also a reference to Angelo Secchi (1818-1878) a pioneering Italian astrophysicist who was one of the first to apply the new technology of photography to recording eclipses. In a similar way we anticipate that SECCHI will provide pioneering observations of CMEs as well as other structures, such as loops, plumes, streamers, comets, etc., using stereo reconstruction techniques.

\author{
W. Deutsch \\ Max-Planck-Institut for Solar System Research, Katlenburg-Lindau, Germany \\ D. Wang $\cdot$ N. Rich \\ Interferometrics, Inc., 13454 Sunrise Valley Drive, Herndon, VA 20171, USA \\ S. Cooper · V. Stephens · G. Maahs \\ HYTEC Inc., 110 Eastgate Drive, Los Alamos, NM 87544, USA \\ R. Baugh · D. McMullin · T. Carter \\ Praxis, Inc., 2550 Huntington Ave. Suite 300, Alexandria, VA 22303, USA
}


The primary objective of the STEREO mission is to understand the CME phenomenon, a phenomenon that was discovered in 1971 and has been observed by coronagraphic instruments on five missions, most recently the ESA/NASA Solar and Heliospheric Mission (SOHO) (see Howard 2006 and references therein). But, this is the first mission whose primary objective is to elucidate the CME itself. The primary objectives of SECCHI are:

- What is the timing of physical properties involved in CME initiation? What are the structures involved in the CME initiation?

- What is the 3-dimensional structure and kinematic properties of CMEs?

- What is the 3-dimensional structure of active regions, coronal loops, helmet streamers, etc.?

- What are the critical forces controlling the propagation of CMEs in the corona and interplanetary medium?

The SECCHI instrument suite consists of five telescopes covering a broad range of fields of view, starting at the solar surface and extending all the way to the interplanetary space between the Sun and Earth. The five telescopes are of three types. The first is an extreme ultraviolet (EUV) imager, EUVI that will image the solar chromosphere and low corona in four emission lines out to $1.7 \mathrm{R}_{\odot}$. The COR1 and COR2 telescopes are the second type and are visible light Lyot coronagraphs with nested fields of view. These two telescopes will image the inner and outer corona from 1.4 to $15 \mathrm{R}_{\odot}$ and were split into two telescopes because of the large radial gradient of coronal brightness in this height range. The third type of telescope extends the coronal imaging from $15 \mathrm{R}_{\odot}$ out to the radius of Earth at $215 \mathrm{R}_{\odot}$. To satisfy the stray light requirement over this field, the task was divided into two wide field heliospheric imagers (HI1 and HI2). Figure 1 gives a summary of the science objectives and how each telescope in the SECCHI suite will contribute. EUVI and the CORs are instruments that have flown on previous missions, but the HI is a new concept.

STEREO provides us with several important new opportunities for CME research, including the following:

\section{SECCHI Exploration of CMEs and the Heliosphere on STEREO \\ -What Configurations of the Corona Lead to a CME? -What Initiates a CME? \\ - What Accelerates CMEs? \\ - How Does a CME Interact With the Heliosphere? - How do CMEs Cause Space Weather Disturbances?}

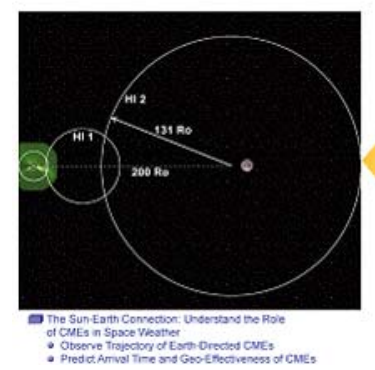

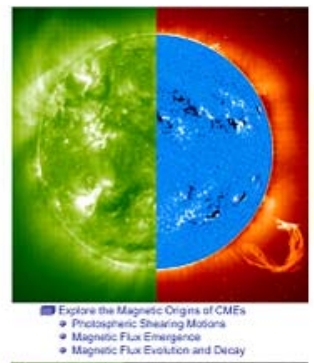

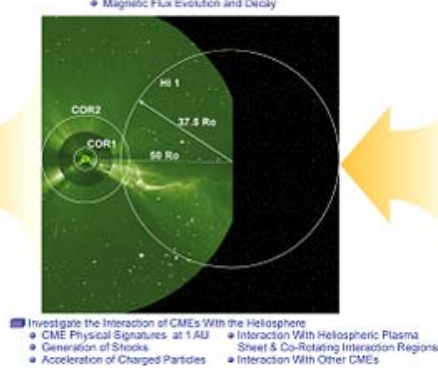

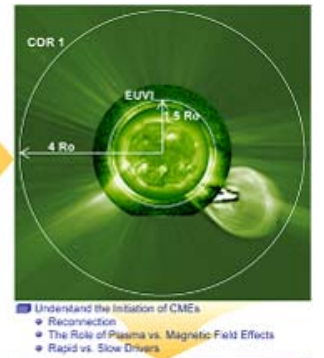

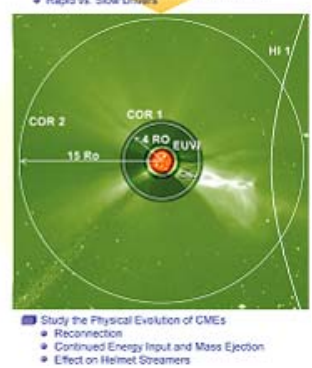

Fig. 1 Summary of SECCHI science objectives 
- The first opportunity to obtain stereographic views of CMEs;

- The first opportunity to observe a CME in interplanetary space at the same time that insitu measurements are made;

- The first opportunity to make simultaneous optical and radio observations of CMEs and shocks;

- The first opportunity to observe geo-effective CMEs along the Sun-Earth line in interplanetary space;

- The first opportunity to detect CMEs in a field of view that includes the Earth.

In this paper we present an overview of the SECCHI instrument-the five main telescopes, the guide telescope (GT), the optical bench for the sun-pointed instruments, the mechanisms, the SECCHI electronics, the CCD detector, housing and control electronics, the flight software and the concept of operations. The hardware is assembled into three packages: (1) the Sun-Centered-Instrument Package (SCIP) which has the EUVI, COR1, COR2, GT and the SCIP camera electronics box (CEB), (2) the Heliospheric Imager (HI), which contains both the HI-1 and HI-2 and the HI-CEB, and (3) the SECCHI electronics box (SEB). The SCIP is located within the spacecraft cylinder at the center of the spacecraft; the $\mathrm{HI}$ is located on the earthward-pointing spacecraft face and the SEB is located within the spacecraft.

\section{Extreme UltraViolet Imager (EUVI)}

\subsection{EUVI Telescope Overview}

The EUVI (see Wülser et al. 2003) observes the chromosphere and low corona in four different EUV emission lines between 17.1 and $30.4 \mathrm{~nm}$. It is a small, normal-incidence telescope with thin metal filters, multilayer coated mirrors, and a back-thinned CCD detector. Figure 2 shows one of the EUVIs during integration at LMSAL. Figure 3 is a cross section through the telescope.

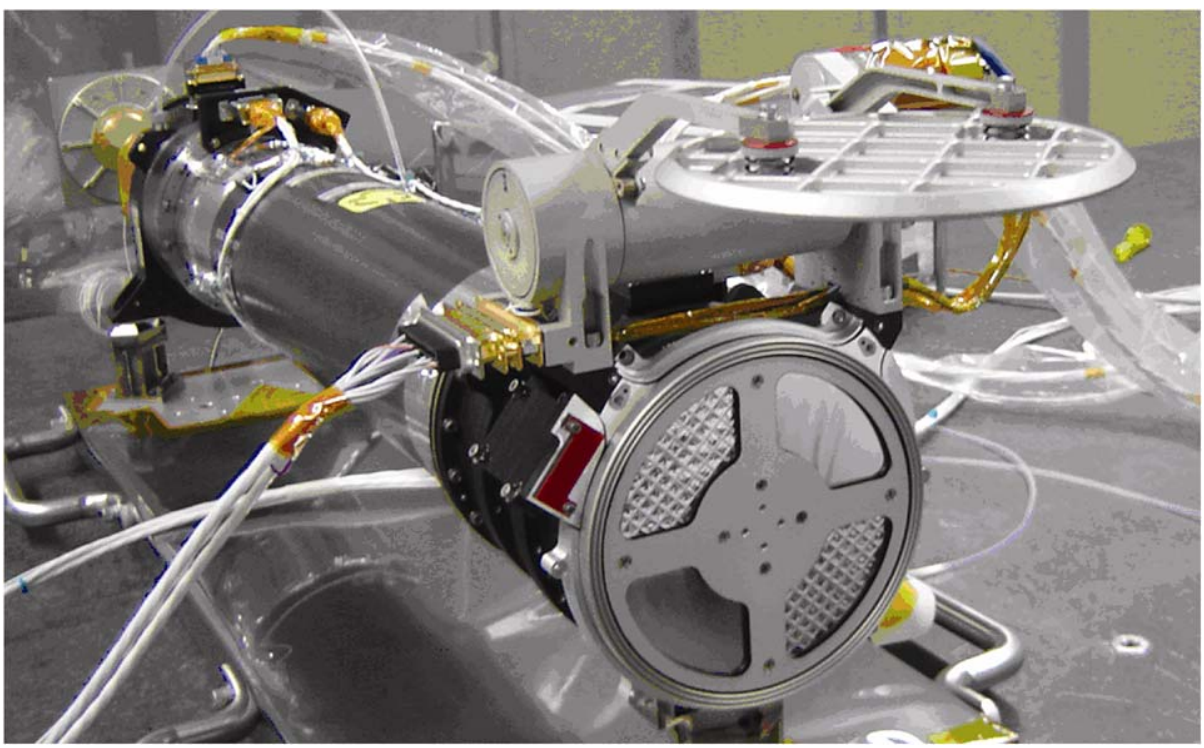

Fig. 2 The EUVI telescope prior to integration into the SECCHI suite. The door is open and reveals the thin $\mathrm{Al}$ entrance filters. The bottom left and top right filters are mesh based filters, the others are polyimide backed supported on a coarse grid 


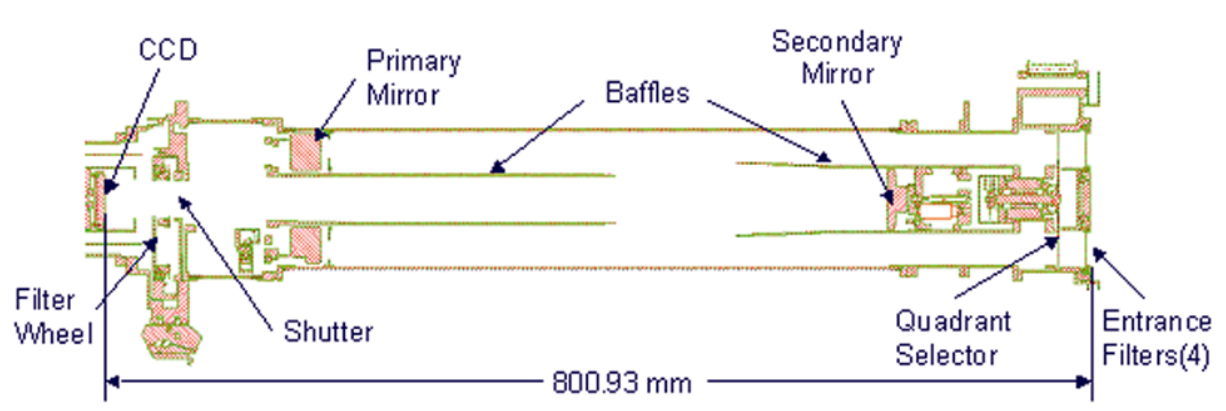

Fig. 3 EUVI telescope cross section

Table 1 EUVI telescope properties

\begin{tabular}{ll}
\hline Instrument type & Normal incidence EUV telescope (Ritchey-Chrétien) \\
Wavelengths & He II $30.4 \mathrm{~nm}$, Fe IX $17.1 \mathrm{~nm}$, Fe XII $19.5 \mathrm{~nm}$, Fe XV $28.4 \mathrm{~nm}$ \\
IR/visible/UV rejection & $>10^{13}$ using thin metal film filters \\
Aperture & $98 \mathrm{~mm}$ at primary mirror \\
Effective focal length & $1750 \mathrm{~mm}$ \\
Field of view & Circular full sun field of view to \pm 1.7 solar radii \\
Spatial scale & $1.6^{\prime \prime}$ pixels \\
Detector & Backside illuminated CCD (e2v CCD42-40), $2048 \times 2048$ pixels \\
Mechanisms & Aperture door, Quadrant selector, Filter wheel, Focal plane shutter \\
Image Stabilization & Active secondary mirror (tip/tilt) \\
& Range: $\pm 7^{\prime \prime}$, jitter attenuation: factor 3 at $10 \mathrm{~Hz}$ \\
\hline
\end{tabular}

EUV radiation enters the telescope through a thin metal film filter of $150 \mathrm{~nm}$ of aluminum. This filter suppresses most of the UV, visible, and IR radiation and keeps the solar heat out of the telescope. During launch, the filter is protected by the front door. The radiation then passes through the quadrant selector to one of the four quadrants of the optics. Each quadrant of the primary and secondary mirror is coated with a narrow-band, multilayer reflective coating, optimized for one of four EUV lines. After bouncing off the primary and secondary mirror, the radiation continues through a filter wheel that has redundant thin-film aluminum filters to remove the remainder of the visible and IR radiation. A rotating blade shutter controls the exposure time. The image is formed on a CCD detector. The main parameters for the EUVI telescope are summarized in Table 1.

\subsection{Optics}

\subsubsection{Optical Design}

The EUVI telescope is a Ritchey-Chrétien system with a secondary mirror magnification of 2.42. This system provides pixel limited resolution across the entire field of view in all four quadrants. The low secondary mirror magnification reduces the telescope's sensitivity to shifts in the mirror separation and eliminates the need for a focus mechanism. The telescope is fully baffled to prevent charged particles entering the front aperture from reaching the CCD. The telescope pupil is located just in front of the primary mirror and is defined by an aperture mask. The baffles and aperture mask have been designed for an unvignetted 


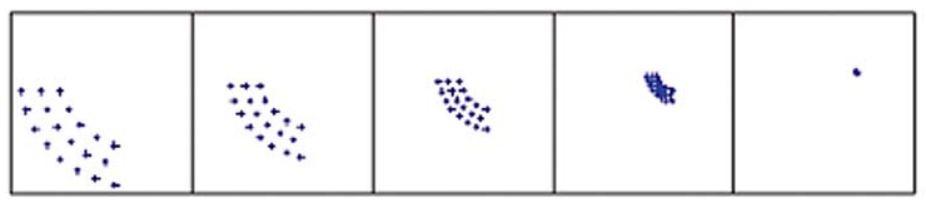

On-Axis

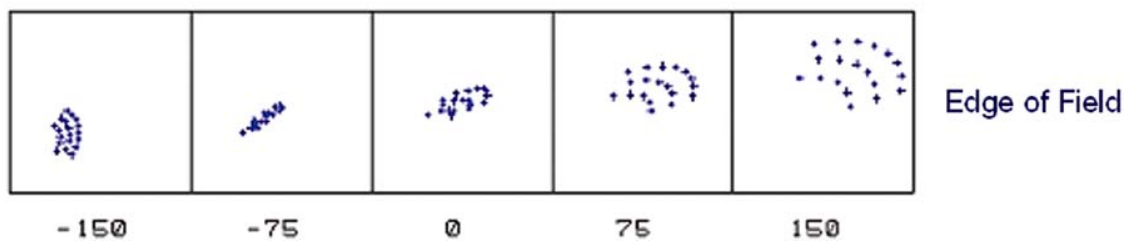

Fig. 4 Ray trace results for a single quadrant and two field angles ( 0 and 27 arcmin) at nominal focus, and up to $150 \mu \mathrm{m}$ inside $(-150)$ and outside (150) of nominal focus. The boxes indicate the size of one CCD pixel

Table 2 Properties of the multilayer coatings

\begin{tabular}{lllll}
\hline Quadrant/channel & $\mathbf{1 7 . 1}$ & $\mathbf{1 9 . 5}$ & $\mathbf{2 8 . 4}$ & $\mathbf{3 0 . 4}$ \\
Center wavelength (two mirror system) & $17.3 \mathrm{~nm}$ & $19.6 \mathrm{~nm}$ & $28.5 \mathrm{~nm}$ & $30.7 \mathrm{~nm}$ \\
$\begin{array}{l}\text { Bandwidth } \\
\text { (measured FWHM for single reflection) }\end{array}$ & $1.4 \mathrm{~nm}$ & $1.6 \mathrm{~nm}$ & $1.9 \mathrm{~nm}$ & $3.0 \mathrm{~nm}$ \\
$\begin{array}{l}\text { Peak reflectivity } \\
\text { (measured, for single reflection) }\end{array}$ & $39 \%$ & $35 \%$ & $15 \%$ & $23 \%$ \\
Coating materials & $\mathrm{MoSi}$ & $\mathrm{MoSi}$ & $\mathrm{MoSi}$, var. spacing & $\mathrm{MoSi}$ \\
\hline
\end{tabular}

field of view to \pm 1.7 solar radii. Figure 4 shows ray trace results for a single quadrant, both on-axis and at the edge of the field, and up to $0.15 \mathrm{~mm}$ inside and outside of nominal focus. The system has a minor amount of field curvature; the nominal focus location is chosen to minimize the aberrations across the field.

\subsubsection{Mirrors}

The EUVI mirrors were figured, polished, and multilayer coated at the Institut d'Optique in Orsay, who also made the mirrors for SOHO/EIT. The Zerodur mirror substrates were first polished to a sphere and superpolished to the required surface roughness. They were then aspherized using an ion beam etching technique that preserves the superpolished properties of the surface. Finally, each quadrant of each mirror was coated with a narrow passband reflective multilayer, optimized for the specific EUV emission to be observed in that quadrant. All coatings consist of MoSi layer pairs using constant layer spacing. The coating for the $28.4 \mathrm{~nm}$ quadrant has a variable Mo/Si thickness ratio for optimum suppression of the nearby $30.4 \mathrm{~nm}$ He II emission line. The other coatings use constant layer spacings. Table 2 summarizes the properties of the coatings (Ravet et al. 2003). 


\subsubsection{Filters}

The EUVI uses thin metal film filters at both the entrance aperture and near the focal plane to suppress undesired UV, visible, and IR radiation. Two types of filters are at the entrance of the telescope (Fig. 2): an aluminum-on-polyimide foil on a coarse nickel grid for the short wavelength quadrants $(17.1$ and $19.5 \mathrm{~nm})$, and a single layer aluminum foil on a fine nickel mesh for the long wavelength quadrants (28.4 and $30.4 \mathrm{~nm})$. Both types of filters use a $150 \mathrm{~nm}$ thick layer of aluminum to reject visible light. The grid-supported aluminum filter is backed with a $70 \mathrm{~nm}$ thick layer of polyimide for strength. The polyimide layer allows the filter to be supported by a coarse grid with $5 \mathrm{~mm}$ line spacing that causes only minimal diffraction at EUV wavelengths. The polyimide transmits only about $50 \%$ of the EUV radiation at the observing wavelengths, but this is not a major concern for the strong lines at 17.1 and $19.5 \mathrm{~nm}$.

The mesh supported filter avoids the absorbing polyimide layer, whose transmission is too low at longer wavelengths, especially for the somewhat weaker line at $28.4 \mathrm{~nm}$. However, the fine mesh, with $0.36 \mathrm{~mm}$ line spacing, causes a noticeable amount of diffraction. Both types of filters have been flown on highly successful experiments: EIT used a plastic reinforced aluminum foil on a nearly identical coarse grid for all wavelengths and TRACE (Handy et al. 1999; Strong et al. 1994) used fine mesh supported filters nearly identical to the ones on the EUVI.

Near the focal plane, a final filter reduces the visible light transmitted by the first filter and by any pinholes that form in the front filter. This final filter is in a filter wheel with four positions, to provide additional protection against pinholes. A third filter wheel position contains two filters in series to mitigate against any catastrophic damage to the entrance filters. The fourth filter slot is left open, which enabled visible light testing on the ground. All filters were manufactured by LUXEL Corporation.

\subsubsection{Aliveness Source}

The EUVI telescope contains blue and violet light emitting diodes (LEDs) for testing and calibration purposes. One set of LEDs is mounted in the spider and illuminates the detector through reflection off the two telescope mirrors. A second set is mounted near the CCD. Photons from the blue LED at $470 \mathrm{~nm}$ have a similar penetration depth in silicon as EUV photons, while photons from the violet LED at $400 \mathrm{~nm}$, which have a shallower penetration depth, provide a diagnostic that is sensitive to CCD surface effects.

\subsection{Mechanical Design}

\subsubsection{Metering Structure}

The EUVI uses a Graphite/Cyanate Ester metering tube as the main telescope structure. The tube stiffness maintains proper alignment of the optical system through launch. The low coefficient of thermal expansion (CTE) in the axial direction minimizes changes in the mirror separation and keeps the telescope in focus throughout the operational temperature range. This eliminates the need for a focus mechanism. The metering tube is lined with an aluminum foil on the inside that acts as a vapor and contamination barrier.

Attached to the front of the metering tube are the secondary mirror spider, the quadrant selector spider, and the entrance chamber with the entrance filters and the interfaces to the aperture door and the forward mount. The spider arms are hollow and incorporate separate vent paths for the secondary mirror tip-tilt mechanism and for the quadrant selector motor. 

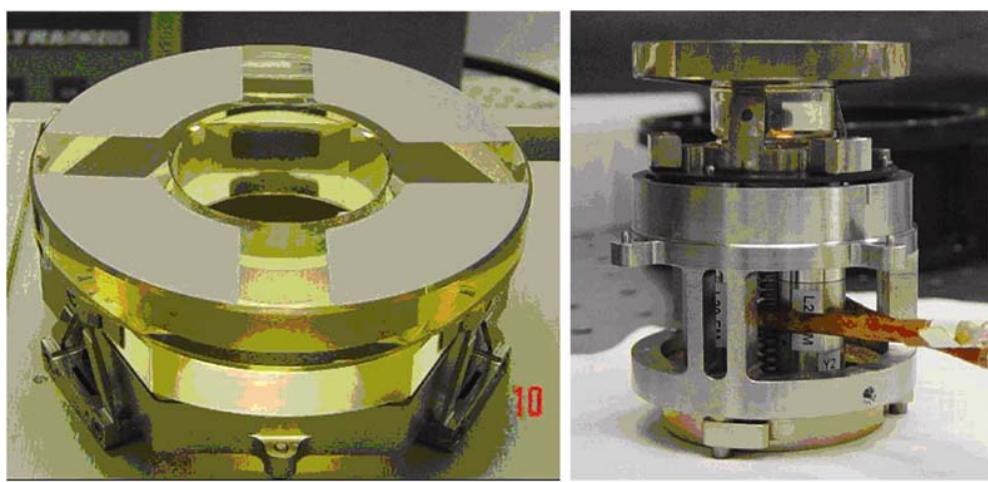

Fig. 5 Left-EUVI primary mirror, coated and mounted. The mirror diameter is $105 \mathrm{~mm}$. The regions between the quadrants are deliberately left uncoated. Right-EUVI secondary mirror and tip-tilt mechanism. The mirror diameter is $48 \mathrm{~mm}$. One of the three PZTs (marked "L2... M") is visible inside the housing

Attached to the aft end of the metering tube are the primary mirror mount and the aft metering structure with the shutter and filter wheel mechanisms, as well as the interfaces to the focal plane assembly and the aft mounts. The aft structure again incorporates separate vent paths for its mechanisms to minimize potential sources of contaminants inside the optical cavity.

\subsubsection{Mirror Mounts}

The EUVI primary mirror and mount are shown in Fig. 5. The mount consists of a hexagonal Titanium ring that interfaces to the mirror substrate via three bi-pod flexures. This arrangement is semi-kinematic: each bi-pod strongly constrains two degrees of freedom, but is relatively flexible in the other four, thus isolating the mirror from thermal stresses in the mount. Interferometric tests showed that temperature changes of up to $22^{\circ} \mathrm{C}$ cause no measurable deformation of the mirror figure. The bi-pods are made of Invar and attach to the Zerodur mirror through bonded Invar mounting pads. This mirror mount is very compact to fit the tight envelope constraints of the EUVI telescope.

The secondary mirror mount with its tip-tilt mechanism is shown in Fig. 5. The mount is a single piece of Invar with three machined fingers that are bonded to the cylindrical base of the Zerodur mirror substrate. The tip-tilt mechanism is very similar to the one on the TRACE telescope. It uses three piezoelectric (PZT) actuators that push against the Invar mount of the mirror. Software in the SECCHI flight electronics processes fine pointing signals from the SECCHI guide telescope and drives the PZT actuators open loop via a simple digitalto-analog converter and low voltage drivers. The tip-tilt range in the EUVI image space is \pm 7 arcseconds, sufficient to accommodate worst case spacecraft jitter.

\subsection{Instrument Response and Calibration}

\subsubsection{Calibration Results}

The EUVI mirrors were calibrated as pairs at the synchrotron of the Institut d'Astrophysique Spatiale in Orsay. The mirrors were arranged in the same geometry as in the EUVI telescope, 

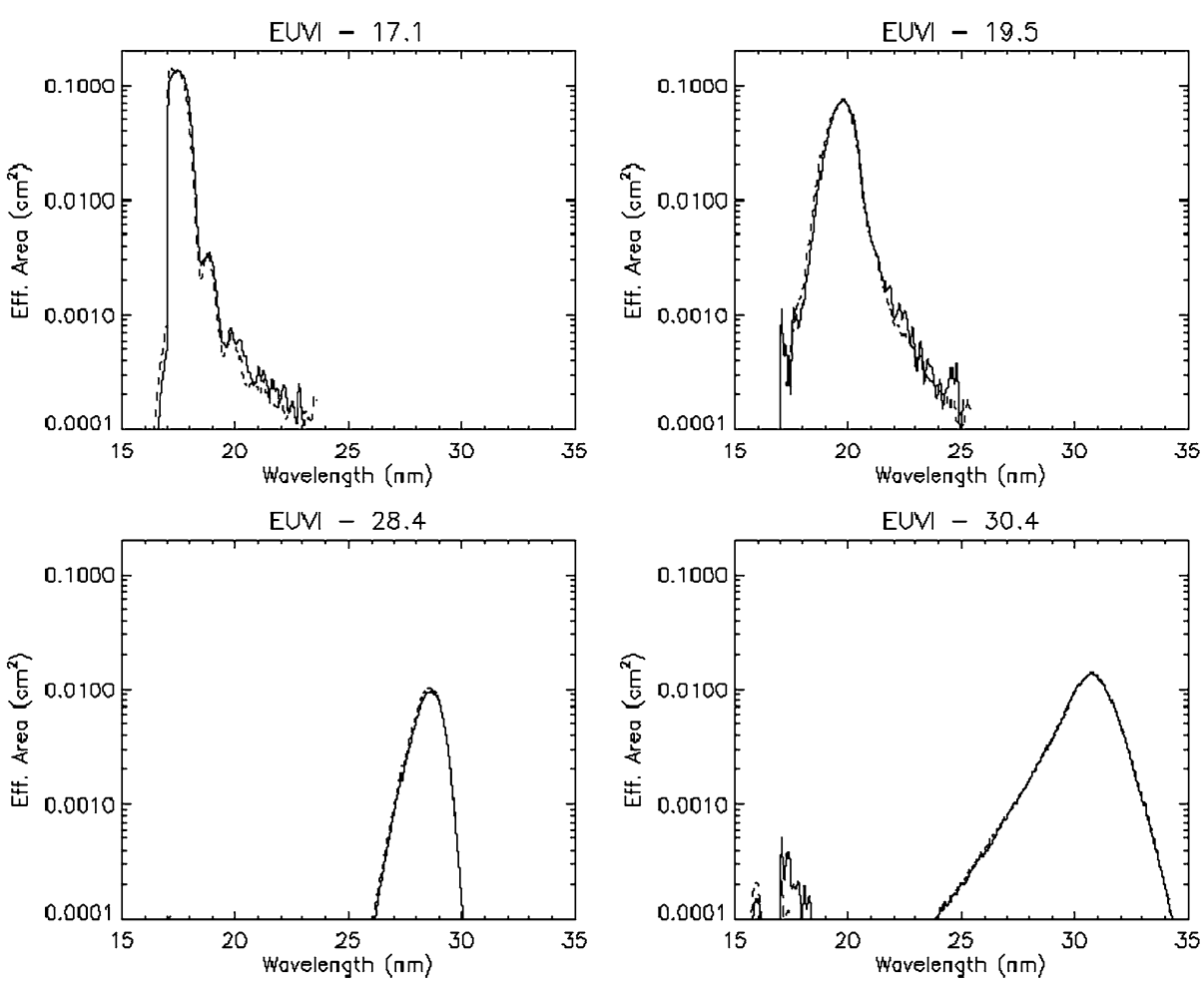

Fig. 6 EUVI effective area. The solid lines are for the EUVI-A, the dashed lines for the EUVI-B

and illuminated with a nearly collimated beam from a monochromator attached to the synchrotron. Each telescope quadrant was measured individually. Wavelength scans were performed with and without the telescope in the beam; the measured ratio provides the absolute total reflectivity of the mirror pairs. Single reflection coating properties are reported in Table 2 . All coatings perform well, both in terms of high reflectivity and proper wavelength of peak reflectivity. The coating for $28.4 \mathrm{~nm}$ is optimized for rejecting the strong He II line at $30.4 \mathrm{~nm}$, which results in a somewhat lower peak reflectivity as expected.

CCDs were calibrated on the NRL beamline at Brookhaven synchrotron and at the LMSAL XUV calibration facility. The entrance and focal plane filters were also calibrated at the LMSAL XUV calibration facility (Windt and Catura 1988). The results of those measurements were used to fit CCD and filter response models. The calibration curves of the individual components were combined to obtain the EUVI effective area as a function of wavelength. The results are shown in Fig. 6. The two telescopes (EUVI-A and EUVI-B) have very similar responses.

\subsubsection{Predicted Response to Solar Phenomena}

Using the calibration results we predict the response of the EUVI to typical solar plasmas. We take typical differential emission measure distributions (DEMs) reported in the literature, predict the resulting solar spectral line emission using the CHIANTI software (Dere et al. 1997; Young et al. 2003), and combine the result with our calibration data. Table 3 summarizes the pixel count rates for selected solar features in the different EUVI channels. 
Table 3 Photon count rates (photons/pixel/second) for some solar features predicted with the CHIANTI code. The numbers for the $30.4 \mathrm{~nm}$ channel have been tripled to adjust for the fact that CHIANTI typically underestimates the He II flux. The first number in each box is for the EUVI-A, the second for the EUVI-B

\begin{tabular}{llll}
\hline Photons/s/pixel & Quiet Sun & Active region & M class flare \\
\hline $17.1 \mathrm{~nm}$ & $92 / 98$ & $954 / 976$ & $25700 / 26700$ \\
$19.5 \mathrm{~nm}$ & $40 / 41$ & $784 / 792$ & $92200 / 101500$ \\
$28.4 \mathrm{~nm}$ & $4 / 4$ & $118 / 130$ & $5540 / 6110$ \\
$30.4 \mathrm{~nm}$ & $30 / 30$ & $428 / 419$ & $18100 / 17800$ \\
\hline
\end{tabular}
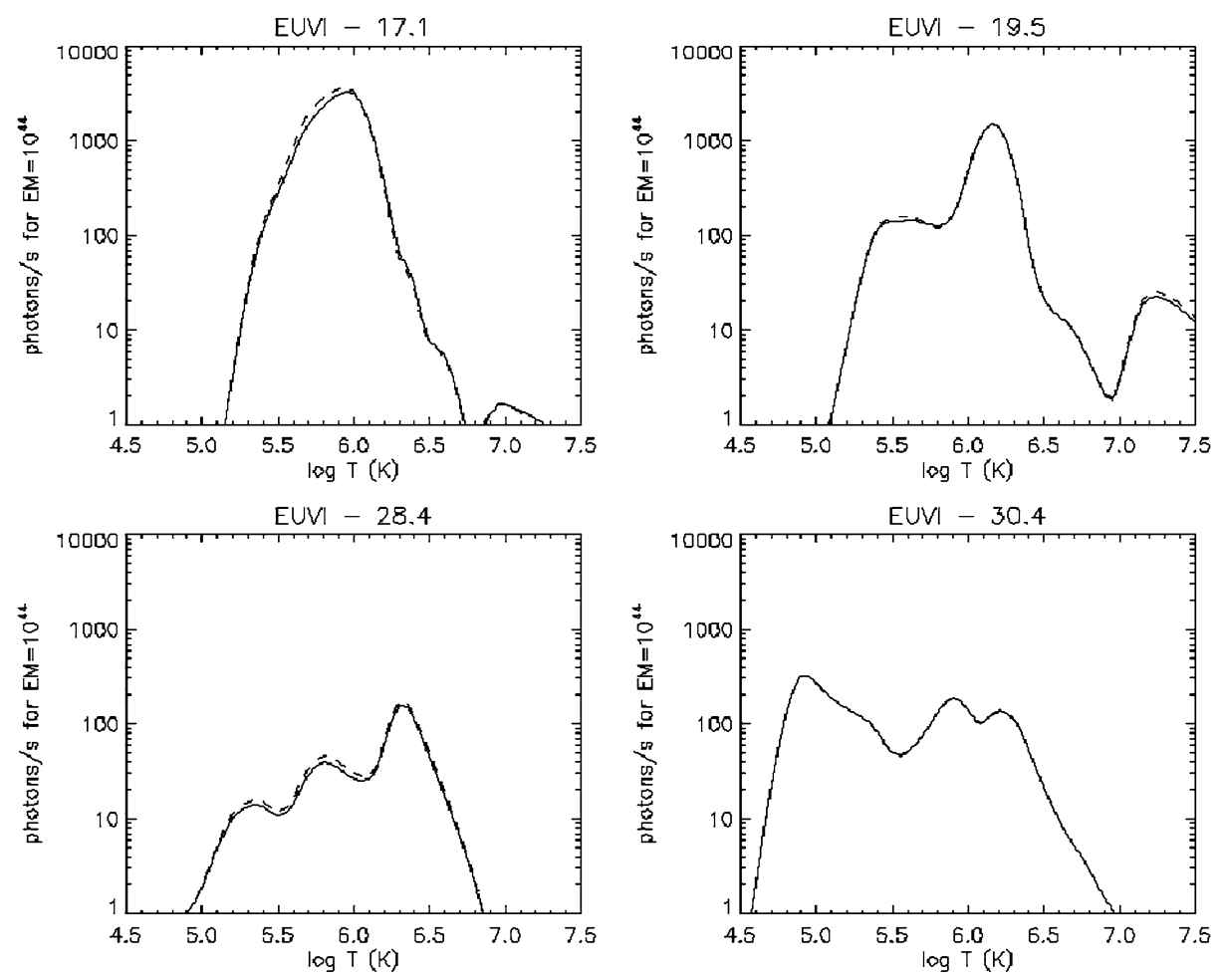

Fig. 7 The response of the EUVI as a function of solar plasma temperature. The solid lines are for the EUVI-A, the dashed lines for the EUVI-B

Note that CHIANTI underestimates the He II flux by typically a factor of three so we have multiplied the prediction by a factor of 3 . Figure 7 shows count rates (in photons/pixel/s) predicted for isothermal plasmas (for an EM of $10^{11} \mathrm{~cm}^{-5}$ ) as a function of plasma temperature.

\section{Inner Coronagraph (COR1)}

\subsection{COR1 Overview}

Like all coronagraphs, COR1 is designed to measure the weak light from the solar corona in the presence of scattered light from the much brighter solar photosphere. It is a classic 
Lyot internally occulting refractive coronagraph (Lyot 1939) which will observe the white light corona from 1.4 to $4 \mathrm{R}_{\odot}$. The COR1 design is the first space-borne internally occulted refractive coronagraph in contrast to the LASCO/C1 (Brueckner et al. 1995), which was an internally occulted reflective design. The optical design, described in the next section, uses the principles articulated by Lyot to eliminate some of the sources of stray light. The internal occultation enables better spatial resolution closer to the limb than an externally occulted design.

The COR1 signal will be dominated by instrumentally scattered light, which must be removed to measure the underlying coronal signal. This stray light cannot be removed by the Lyot principles but is largely unpolarized and therefore can be eliminated or at least greatly reduced by making polarized observations in three states of linear polarization and calculating the polarized brightness $(\mathrm{pB})$. To achieve this separation, we must have a high enough signal to noise ratio, even in the presence of the large scattered light noise. This is partly achieved by performing on-board binning of the pixels.

\subsection{Optical Layout}

Figure 8 shows the opto-mechanical layout of the COR1 instrument. Sunlight enters through the front aperture, where the objective lens focuses the solar image onto the occulter. To keep scattering to a minimum, a singlet lens is used for the objective, made of radiation hardened BK7-G18 glass.

The occulter is mounted on a stem mounted at the center of the field lens (Fig. 9). The tip of the occulter is cone shaped, to direct the sunlight into a light trap, which surrounds the occulter. The radius was chosen to block all wavelengths $(350-1100 \mathrm{~nm})$ out to a radius of $1.1 \mathrm{R}_{\odot}$ at orbital perigee. At the design wavelength of $656 \mathrm{~nm}$, the solar image is completely occulted out to $1.30 \mathrm{R}_{\odot}$, and partially vignetted out to $1.64 \mathrm{R}_{\odot}$. (When combined with the

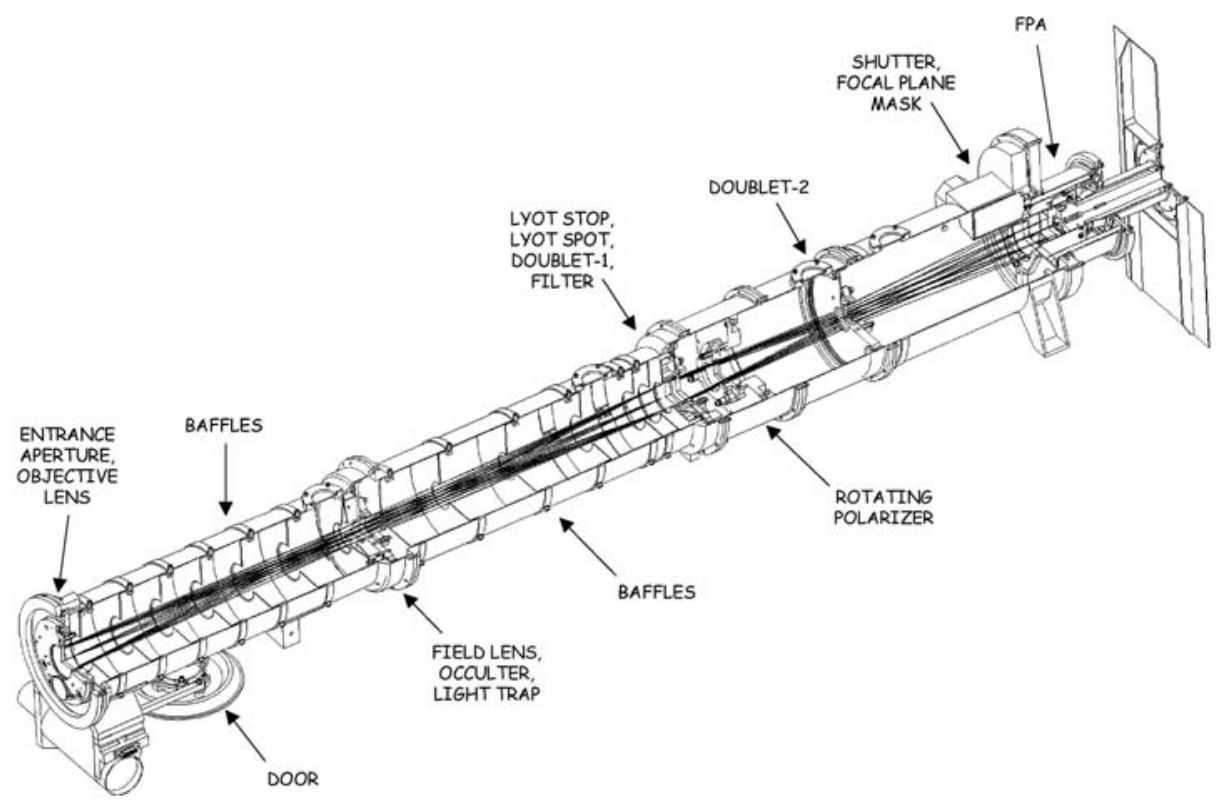

Fig. 8 Layout of the COR1 instrument package 

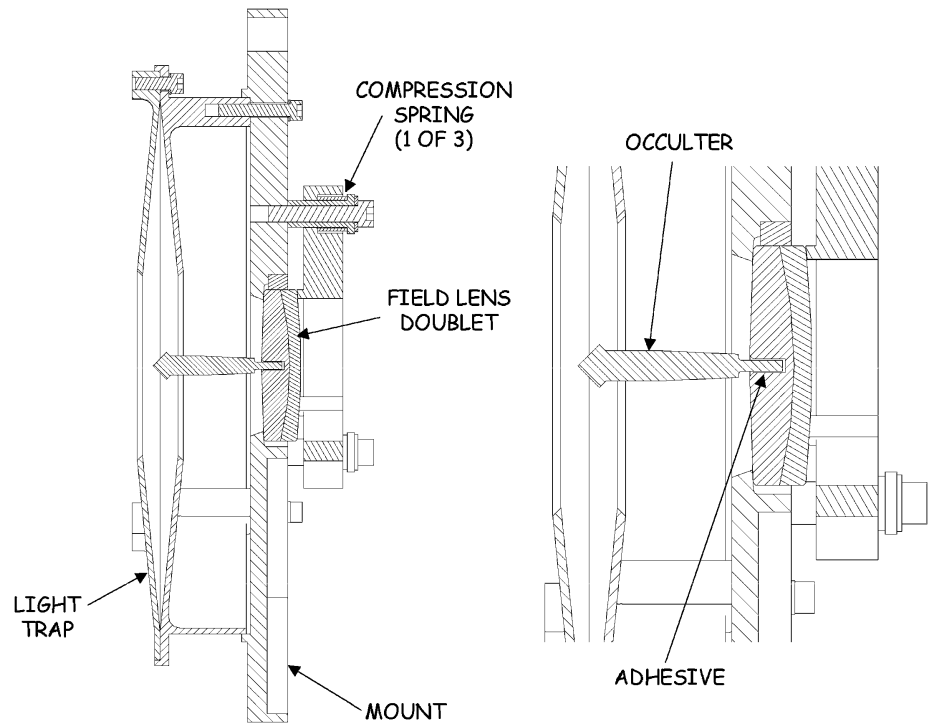

Fig. 9 The conical occulter mounted at the center of the field lens, and surrounded by the light trap. Hashed regions show the presence of material at the central plane in this cutaway view

focal plane mask discussed below, these numbers become 1.4 and $1.9 \mathrm{R}_{\odot}$ respectively.) The wedge-shaped design of the light trap ensures that any ray entering it must reflect many times, so that the light will be absorbed before it can find its way out again.

Two doublet lenses are used to focus the coronal image onto the CCD detector. The first, a positive power achromat, is placed immediately behind the Lyot stop, while the second, a negative power achromat, is placed further down the optical path. Together, these act as a telephoto-lens system, focusing the coronal image onto the detector plane, while maintaining diffraction-limited resolution. A bandpass filter $22.5 \mathrm{~nm}$ wide, centered on the $\mathrm{H} \alpha$ line at $656 \mathrm{~nm}$, is placed just behind the first doublet. Thus, the Lyot stop, spot, first doublet, and bandpass filter form a single optical assembly.

A linear polarizer on a hollow core motor rotational stage is located between the two doublets. The polarizing material is Corning's Polarcor, chosen for its uniformity. The placement of the polarizer was chosen to be as close to the first doublet as possible within the dimension constraints of the largest diameter piece of Polarcor that can be obtained $(33 \mathrm{~mm}$ clear aperture). Normal operations call for three sequential images to be taken with polarizations of 0 degrees and \pm 60 degrees, to extract the polarized brightness.

\subsection{Stray Light Suppression}

The primary photospheric light suppression mechanisms in COR1 are those described by Lyot (1939) - (1) the objective lens to occulter imaging system, (2) the aperture stop to field lens to Lyot stop imaging system and (3) a Lyot spot. A final stray light suppression technique is the inclusion of a focal plane mask.

The singlet objective lens only focuses the solar image accurately at a single wavelength. Thus only a single wavelength of the photosphere is blocked by the internal occulter. A narrow band filter defines the passband of the instrument, which matches the wavelength at the primary focus of the objective lens. Rather than relying on the out-of-band rejection of 
the filter, the primary suppression mechanisms are designed to work over the full sensitivity wavelength band of the instrument.

The solar image from the objective will be chromatically aberrated, so the occulter must be sized to block all the solar photospheric light from the near UV to infrared (350$1100 \mathrm{~nm}$ ). The cut-on at $350 \mathrm{~nm}$ is set by the transmission of the BK7-G18 glass in the objective lens, and the cut-off at $1100 \mathrm{~nm}$ is set by the band gap of the silicon detector. Subsequent lenses in the optical train balance the chromatic aberration from the objective. The narrow bandpass of the instrument also minimizes the effect of chromatic aberration in the final image.

Diffracted light from the edge of the front entrance aperture is focused onto a Lyot stop by the field lens and removed. This eliminates the largest source of stray light in the system. Additional stray light rejection is accomplished by placing baffles at various points between the front aperture and Lyot stop. A Lyot spot is also glued to the front surface of the doublet lens immediately behind the Lyot stop, to remove ghosting from the objective lens.

A focal plane mask is located between the shutter and the focal plane detector, and is used to remove diffracted light from the edge of the occulter. This circular mask, mounted on a plane of BK7 glass, is sized to be slightly larger than the occulter image at that location. Without the mask, the image on the detector would be dominated by bright rings at the umbral and penumbral edges of the occulter shadow. The addition of the focal plane mask removes these bright rings. The focal plane assembly is discussed in Sect. 9.

\subsection{Mechanical and Thermal Design}

The COR1 mechanical structure is designed as a series of tube sections (Fig. 8), which are bolted and pinned together for stability. The individual optics are aligned, mounted, and pinned within these tube sections. COR1 assembly starts from the front tube section, with the objective, occulter, light trap, and field lens, and then each subsequent section is added, together with its associated optical or mechanical components. Because the individual tube sections are pinned together, sections can be taken off and back on again without changing the optical alignment.

Three mechanisms are included in the COR1 instrument package. At the front of the instrument is a door, to protect the instrument before and during launch, and during spacecraft maneuvers. On the front of this door is a diffuser so that the operation of the instrument can be tested when the door is closed, and to provide a flat-field calibration signal. The diffuser is sized to completely illuminate the objective over all angles within the field-of-view.

The other two mechanisms are the hollow core motor to rotate the linear polarizer, and a rotating blade shutter mounted just in front of the focal plane detector assembly, both supplied by Lockheed Martin. All the mechanisms, together with the focal plane CCD detector, will be operated from a centralized control system for all the SECCHI instruments.

Because the two STEREO spacecraft are in elliptical orbits about the Sun, the COR1 instruments will experience considerable variation in solar load, from 1264-1769 and 1068$1482 \mathrm{~W} / \mathrm{m}^{2}$ for the Ahead and Behind spacecraft respectively. When these loads are combined with the modeled changes in the material thermal properties from beginning to end of life, and with the most extreme differences in the thermal loads from the surrounding structure, the worst-case temperature variation in the COR1 instrument is from 2.5 to $30^{\circ} \mathrm{C}$. There's also an axial gradient in temperature from the front to the back of the instrument, varying from $3^{\circ} \mathrm{C}$ in the cold case, to $7^{\circ} \mathrm{C}$ in the hot case. Strategically placed software controlled proportional heaters with programmable set points, are used to keep the instrument 
within the $0-40^{\circ} \mathrm{C}$ operational temperature range. There are also survival heaters on mechanical thermostat control to keep the instrument within the -20 to $+55^{\circ} \mathrm{C}$ non-operational range.

Specialized composite coatings of oxides over silver are used to help manage the intense solar fluxes which COR1 will be experiencing. The oxide coatings are deposited onto many of the exposed surfaces around the aperture area, such as the objective lens holder assembly and door assemblies. This coating exhibits very low solar absorbtivities, is very stable, and has relatively high IR emissivity values depending on the thickness of the deposited oxide layers. Silver teflon is used for the front layer of the multilayer insulation. The majority of the solar load collected by the front objective is concentrated on the occulter tip (Fig. 9). In the worst-case analysis, the tip can reach a temperature of $125^{\circ} \mathrm{C}$. This tip is made of titanium, and is diamond-turned to direct the sunlight into the light trap. It is coated with a Goddard composite silver coating for high reflectivity. The occulter shaft is coated with black nickel to radiate away the heat. A thin cross-section titanium shaft is used to thermally isolate the occulter from the field lens.

\subsubsection{Calibration and Performance Results}

Before building the COR1 flight units, an extensive test program was carried out on a series of breadboards and engineering test units, using the Vacuum Tunnel Facility at the National Center for Atmospheric Research in Boulder, Colorado (Thompson et al. 2003). These tests were used to demonstrate the instrument concept, and to improve the design for flight. One result of this testing was the addition of a focal plane mask to remove diffracted light from the edge of the occulter.

Characterization of the COR1 flight units was carried out in a similar vacuum tank facility at the Naval Research Laboratory in Washington, DC. The instrument was installed in a large vacuum chamber within a class 10,000 cleanroom. A long vacuum tunnel extended out from one end of the vacuum chamber to a window, where an entrance aperture was mounted to simulate the size of the Sun at perigee. The total distance between the source aperture and the COR1 front aperture was 11 meters. A partially collimated Xenon arc lamp was used to illuminate the source aperture, with the degree of collimation chosen to direct the light onto the COR1 objective and heat shield. Different aperture sizes were used for the Ahead and Behind instruments, because of the different orbital perigees of the two spacecraft.

As well as stray light, this vacuum tunnel facility was also used to measure other performance characteristics, such as resolution, polarization, and photometric response. The photometric calibration of the instrument was measured by illuminating the aperture at the end of the tank with a diffuse unpolarized light source of known intensity. Placing a sheet polarizer in front of this source allowed us to test the polarization response. Focus was tested by projecting an Air Force 1951 resolution test target through a collimator onto the instrument. The instrumental flat field was tested using both the diffuser mounted in the door, and with an external double-opal source mounted in front of the instrument, with identical results.

The vignetting function and flat field response of the instrument is demonstrated in Fig. 10. The field is unvignetted except for a small area around the edge of the occulter, and near the field stop in the corners of the image. (The dim spot in the center of the occulter shadow is caused by scattering within the instrument.) Only the Ahead data are shown-the Behind response is virtually identical.

Figure 11 shows the measured COR1 scattered light performance for the Ahead and Behind instruments. The average radial profile is well below $10^{-6} \mathrm{~B} / \mathrm{B}_{\odot}$ for both instruments. 
Fig. 10 Flat field response of the COR1 Ahead instrument
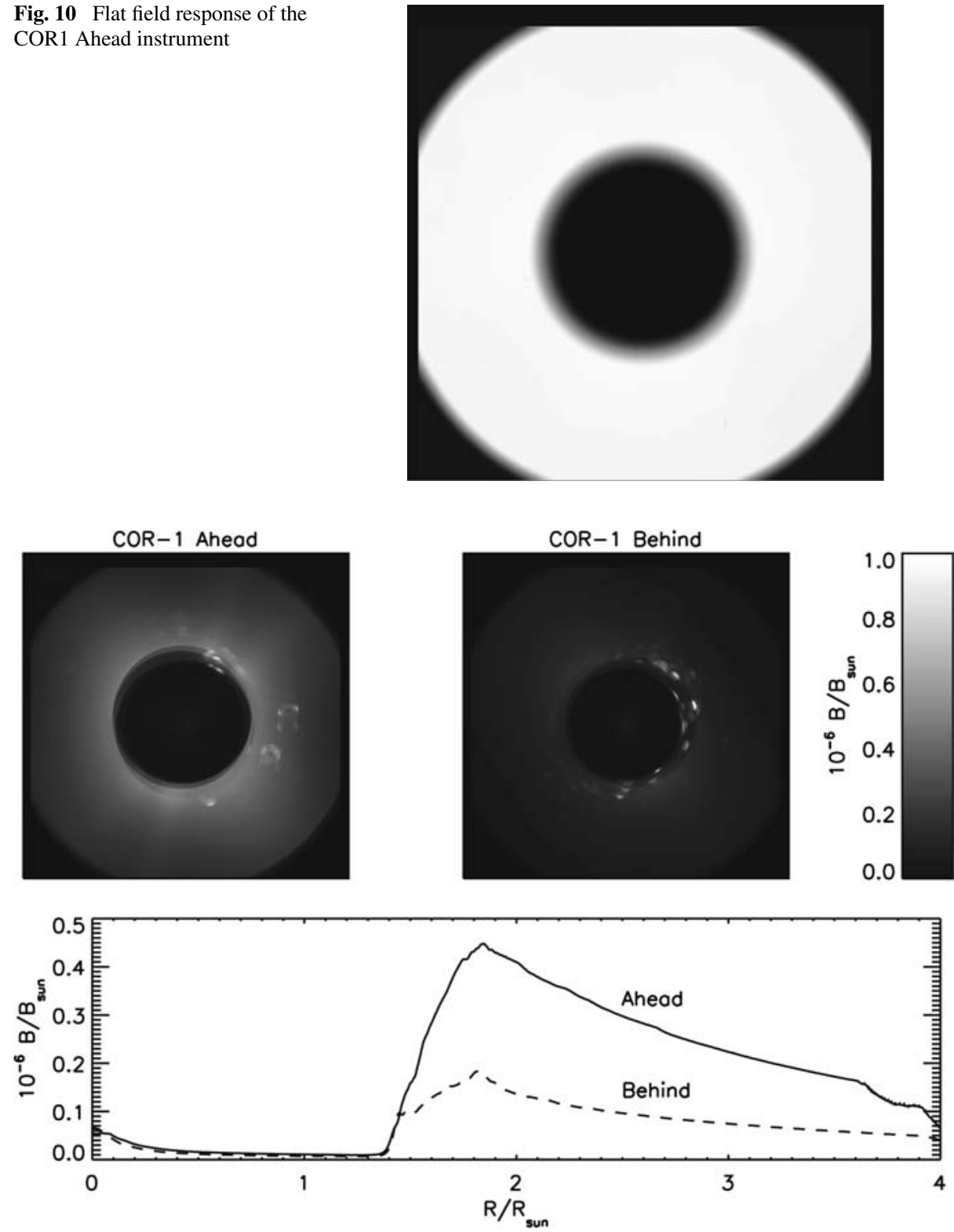

Fig. 11 Measured scattered light images and average radial profiles for the COR1 Ahead (solid) and Behind (dashed) instruments

There are discrete ring-shaped areas of increased brightness, which can climb to as high as $1.4 \times 10^{-6} \mathrm{~B} / \mathrm{B}_{\odot}$ for the Behind instrument. It has been determined that these are caused by features on the front surface of the field lens.

Some contamination was found on the COR1 Behind objective lens after thermal vacuum testing of the STEREO spacecraft. The objective has been cleaned and re-installed, and may no longer have the performance shown in Fig. 11. 


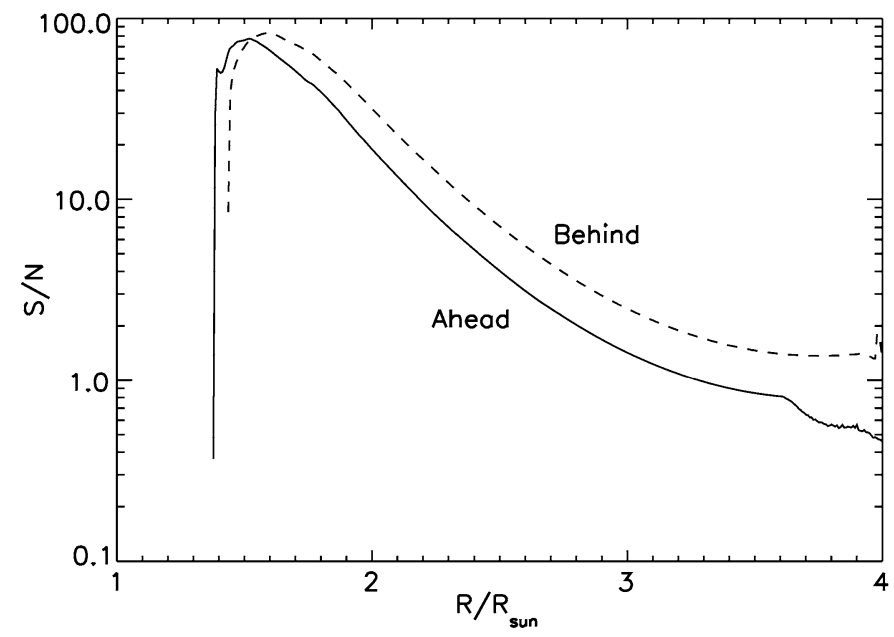

Fig. 12 Estimated signal-to-noise ratios for a modeled K-corona for an exposure time of $1 \mathrm{~s}$, with $2 \times 2$ pixel binning

Table 4 COR1 performance characteristics

\begin{tabular}{llll}
\hline Parameter & Units & Ahead & Behind \\
\hline Pixel size, full resolution & arcsec & 3.75 & 3.75 \\
Pixel size, $2 \times 2$ binned & arcsec & 7.5 & 7.5 \\
RMS spot size (design) & arcsec & 4.17 & 4.17 \\
Planned exposure time & $\mathrm{s}$ & 1 & 1 \\
Polarizer attenuation & - & $10^{-4}$ & $10^{-4}$ \\
Photometric response & $\mathrm{B} \odot / \mathrm{DN}$ & $7.1 \times 10^{-11}$ & $5.95 \times 10^{-11}$ \\
Time to complete pB sequence & $\mathrm{s}$ & 11 & 11 \\
Image sequence cadence & $\min$ & 8 & 8 \\
\hline
\end{tabular}

Combining the data from Figs. 10 and 11, together with a model of the K corona polarized brightness, allows one to estimate the signal-to-noise ratios that will be seen during flight. The results are shown in Fig. 12 for an exposure time of 1 second. The coronal model used is based on Gibson (1973), is valid between 1.4 and 4 solar radii and has the functional form:

$$
\log _{10}(\mathrm{pB})=-2.65682-3.55169\left(\mathrm{R} / \mathrm{R}_{\odot}\right)+0.459870\left(\mathrm{R} / \mathrm{R}_{\odot}\right)^{2} .
$$

The overall performance characteristics of the COR1 instruments are summarized in Table 4. In order to increase the Signal to Noise Ratio, the 3.75 arcsec square pixels will be summed together into $2 \times 2$ bins, to form larger pixels $7.5 \operatorname{arcsec}$ on a side. The design goal for the root-mean-square spot size on the detector was chosen to be $15 \mu \mathrm{m}(4.17 \mathrm{arcsec})$, somewhat larger than the $13.5 \mu \mathrm{m}$ size of a single CCD pixel, but well within the $27 \mu \mathrm{m}$ size of a $2 \times 2$ binned pixel. The largest contribution to the optical error budget comes from thermal changes on orbit, which can be as large as $12 \mu \mathrm{m}$.

The three images in a polarization sequence will be taken as quickly as possible, within $10 \mathrm{~s}$, so that changes due to evolution of the corona will be minimized. Each set of three im- 
ages makes up a complete observation, and the cadence of observations is the time between one three-image set and the next.

\section{Outer Coronagraph (COR2)}

Like the COR1, the SECCHI outer coronagraph, COR2, observes the weak coronal signal in visible light. It is an externally occulted Lyot coronagraph and derives its heritage from the highly successful LASCO C2 and C3 coronagraphs aboard SOHO (Brueckner et al. 1995). The externally occulted design shields the objective lens from direct sunlight, enabling a lower stray light level than COR1 thus achieving observations to further distances from the Sun. It is very complementary to COR1, which observes closer to the Sun.

The primary requirement on the COR2 was to observe CMEs in polarization brightness (pB) with a 15 arc second per pixel spatial resolution. To obtain $\mathrm{pB}$ images, a sequence of 3 linearly polarized images is taken. In order to minimize the smear caused by the CME motion during the polarization image sequence, the sequence of 3 images need to be acquired in a short time. A moderately fast CME moving at the speed of $750 \mathrm{~km} / \mathrm{s}$ would traverse a COR2 pixel $(15$ arcsec) in 15 seconds. This was the time criterion that was established. CMEs moving faster than this can still be accommodated, by binning the image down (on the ground) until the motion is within one pixel.

\subsection{Optical Design}

Solar coronal radiation enters the coronagraph through the A0 aperture (see Fig. 13). A three-disk external occulter shades the objective lens from direct solar radiation and creates a deep shadow at the objective lens aperture (A1). A heat rejection mirror reflects incident solar radiation back through the entrance aperture.

The optical design uses three lens groups, a spectral filter, and a polarizer. The objective lens group (O1) images the external occulter onto the internal occulter and the field lens group (O2) images the A1 aperture onto the A3 aperture (Lyot stop). The O1 also creates an image of the A0 onto A2 (the A0 image stop). The interior occulter and aperture stops capture the brightly illuminated edges of the external occulter and the A0 and A1 apertures. The internal occulter is sized to be slightly larger than the image of the third disk of the external occulter, and the Lyot stop is sized to be slightly smaller than the image of the A1 aperture. These two elements define the inner edge of the coronagraph field of view at a field angle corresponding to an altitude of $2.5 \mathrm{R}_{\odot}$ in the corona at the nominal perihelion distance of each of the STEREO spacecraft. The outer limit of the field of view is determined by the field stop, which is located in the plane of the coronal image formed by the $\mathrm{O} 1$ lens group. The field of view of COR 2 is $\pm 4^{\circ}$, corresponding to a height of $15 \mathrm{R}_{\odot}$ in the corona at $1 \mathrm{AU}$. The third lens group (O3) forms an image of the coronal scene in the focal plane. The optical materials used in the lenses are BK7, LAK10, and SF10. All of these materials have extensive space flight heritage. The spectral filter transmits from $650 \mathrm{~nm}$ to $750 \mathrm{~nm}$ (FWHM). This bandpass optimizes instrument throughput and image quality. The Polarcor polarizer is mounted in a hollow-core motor mechanism (see Sect. 8.5) that rotates the polarizer to various angles.

Adequate K-corona signal detection in the faint outer portion of the field of view requires high exposure and minimal scattered light production at $\mathrm{O} 1$ and $\mathrm{O} 2$. High levels of diffracted light originating at the external occulter and localized in the final image near the $2.5 \mathrm{R}_{\odot}$ 


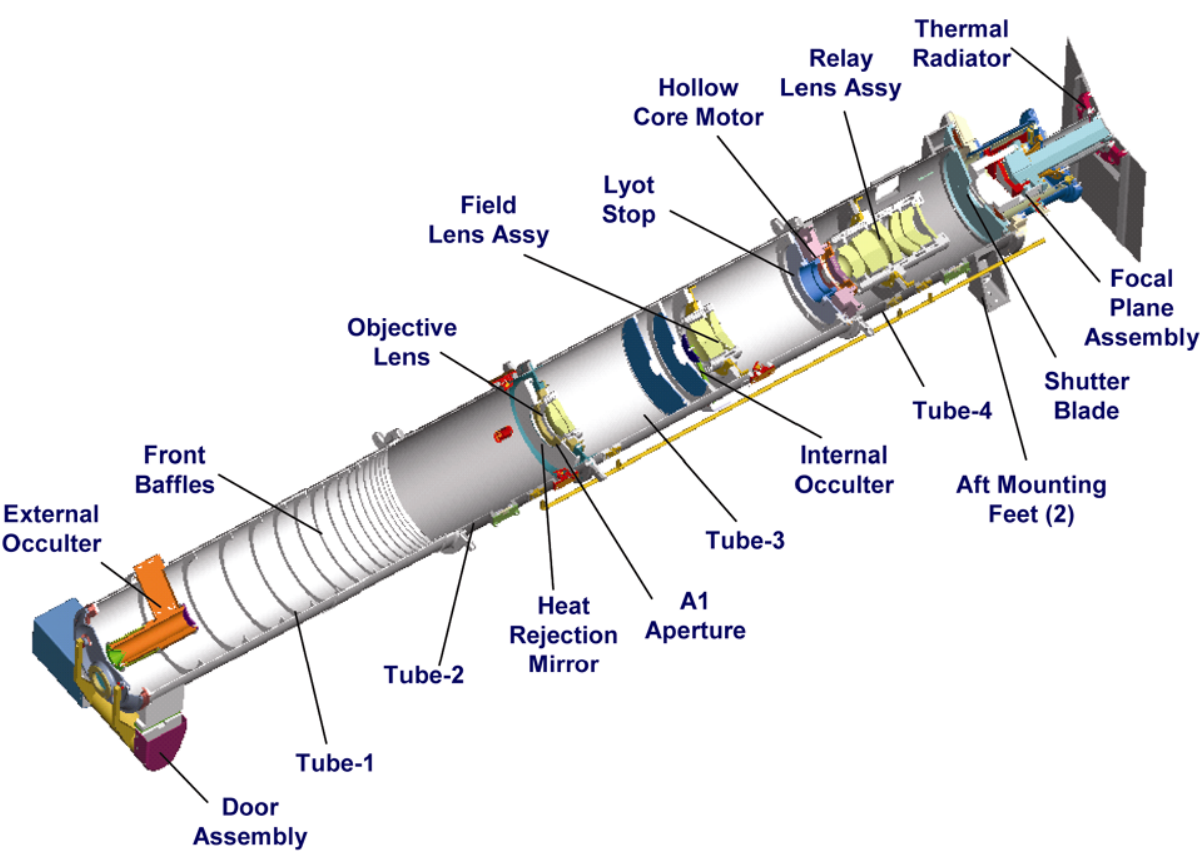

Fig. 13 COR2 mechanical layout

inner field of view cut off would both saturate the CCD before an adequately high exposure is achieved and increase scattered light production by $\mathrm{O} 1$ and $\mathrm{O} 2$. A related concern is the need to accommodate the expected COR1/COR2 bore sight offset and spacecraft jitter. We addressed these issues by paying particular attention to external occulter diffraction. Two diffraction models were developed and validated with laboratory tests. The models allow diffracted light level to be calculated accurately and minimized for a given inner field of view cutoff by adjustment of the diameters of the first two disks of a three-disk occulter system.

In addition to the diffraction modeling and testing, we also paid close attention to another source of instrumental background in the design phase: ghost images of the bright diffraction sourcing at the external occulter. Ghost images are formed by internal reflections of light from the bright edge of the final external occulter disk in the coronagraph, primarily in the $\mathrm{O} 1$ lens and secondarily by reflections from the CCD back toward the lens system. By selecting specific lens surface radii in the optical design phase, we were able to achieve an optical design that confined all significant ghost images present at the focal plane to regions outside the CCD photosensitive area.

The requirement of obtaining a polarization sequence within 15 seconds demanded an optical design that had high light gathering power in comparison to LASCO. Three techniques were used: (1) a shorter exposure time was achieved by using a CCD with a higher quantum efficiency ( $80 \%$ vs. $36 \%$ ) (2) the light gathering power was increased by using a $34 \mathrm{~mm} \mathrm{~A} 1$ aperture rather than $20 \mathrm{~mm}(\mathrm{C} 2)$ or $9 \mathrm{~mm}$ (C3) apertures, and (3) Polarcor polarizing glass was substituted for Polaroid plastic sheet in the linear polarization analyzer which provides better matching of the solar spectral distribution peak to the CCD spectral responsivity. 
The need for C2-like spatial resolution in combination with the $15 \mathrm{R}_{\odot}$ field of view (C2 field limit was $7 \mathrm{R}_{\odot}$ ) was accomplished in part with a $2 \mathrm{k} \times 2 \mathrm{k}$ CCD. The $f$-ratio was set to $f / 5.6$ to avoid a substantial increase in instrument length that would be required to accommodate the larger A1 at the same $f$-ratios used for $\mathrm{C} 3(f / 9.3)$ and $\mathrm{C} 2$ $(f / 20)$. Because the large A1 aperture, wide field angle and high spatial resolution placed a burden on the design of the lens system, particularly the $\mathrm{O} 2$ lens group, the assembly of the lens systems had tight tolerances, which was accomplished by precision potting techniques.

\subsection{Mechanical and Thermal Design}

The COR2 mechanical layout is shown in Fig. 13. The mechanical structure consists of a series of circular cross-section tubes. The tubes are mechanically aligned, match drilled, and pinned to allow for repeatability in assembly. There are a total of four primary tubes for mounting the optics and the FPA (see Sect. 9.2) tube section. The tubes are fabricated from 6061-T6 aluminum. The COR2 instrument interfaces with the SCIP optical bench via a kinematic mount system comprising of three titanium flexures. The fundamental frequency of the structure with the kinematic mounts is $92 \mathrm{~Hz}$. The COR2 instrument weighs approximately $11 \mathrm{~kg}$ and is $1.4 \mathrm{~m}$ long.

The first tube section, Tube 1, supports the door assembly, the A0 aperture, and the external occulter subassembly. Tube 1 is machined with internal baffles to reduce stray light entering A1. The tube incorporates a stiffening ring locally where it interfaces the front flexure for additional stiffness. The external occulter subassembly is cantilevered off the tube wall with a single pylon to reduce diffraction. The occulter system consists of three highly precision-machined disks mounted on a spool. Tube 2 serves as additional metering length for the coronagraph. The objective lens assembly is integrated at the interface between Tubes 2 and 3. The assembly consists of the heat rejection mirror, the A1 stop, and the objective lens set. Tube 3 supports two additional apertures, namely the coronal image and A0 image stops, and the field lens assembly. The internal occulter and the Lyot stop is also mounted to Tube 3 .

Tube 4 supports the hollow core motor, the relay lens set, the shutter mechanism, and the FPA. The mechanisms are described in Sect. 7 and the FPA and CCD in Sect. 9.

The COR2 thermal design is similar to COR 1 in that it uses a slightly cold biased design. A mirror attached to the bulkhead between Tubes 2 and 3 reflects the solar disk radiation coming through the A0 entrance aperture back through the A0. MLI (multi-layer insulation) blankets from the SCIP closeout insulate the instrument from its surroundings. The instrument temperature is maintained using a combination of thermostatically controlled survival heaters and software controlled operational heaters. The structural tube surfaces are treated with an inorganic black anodize. This surface finish provides an optical black and increases thermal coupling within the instrument, reducing thermal gradients.

When the aperture door opens, some of the components comprising the front end of the instrument are exposed to the solar environment. Structural surfaces exposed to direct sunlight are coated with a silver composite coating (CCAG) to prevent overheating. All other surfaces are black anodized to minimize scattered light. The heat rejection mirror, mounted in front of the objective lens, reflects the incident solar radiation back through the A0 aperture and into space. 


\subsection{Calibration and Performance Results}

The COR2 calibration plan involved testing at both the component and telescope levels. The properties of the linear polarizers were measured in the lab and the two best polarizers were chosen for the flight instruments. The bandpass filters were manufactured and measured by Andover. The bandpass response was verified at NRL and again the best filters were used in the flight instruments. The most extensive component-level testing was performed on the various lens sets of the COR2 optical train. A breadboard version of the instrument was constructed to mount the 3 lens groups and to allow us to verify the optical prescription. With the aid of an Air Force resolution target, we were able to measure the MTF at various field angles and choose the lens group combinations with the best imaging performance.

The flight unit performance was measured at NRL's Solar Coronagraph Optical Test Chamber (SCOTCH) facility (Morrill et al. 2006). The same facility was used in a similar configuration for the COR1 calibration (Sect. 3.4.1). The instrument vignetting function and spectral response were measured in air. The stray light levels, image quality and absolute photometric calibration were measured under vacuum. The tests were based on similar tests for the SOHO/LASCO coronagraphs.

The COR2 vignetting pattern for the Ahead telescope is shown in Fig. 14. The image is vignetted throughout the field of view reaching a minimum of $20 \%$ at about $10 \mathrm{R}_{\odot}$ before increasing again towards the edge. The vignetting around the occulter pylon is only about 40-50\%, which does not impede imaging and therefore the pylon will be invisible in the images similarly to LASCO/C2.

The stray light tests (Fig. 15) showed that the instrument performance exceeds the design requirements and will allow us very detailed imaging of the extended corona. The image on the left of Fig. 15 was taken during the stray light test and shows back-reflections onto baffles in the chamber. The plot on the right is the intensity from the center outward to the lower right corner of the image. It passes through one of the low-scatter surfaces (vel-black). The effectiveness of the vel-black is seen immediately in the contrast to the neighboring region which is light being reflected onto the end of the chamber. On the plot is shown the intensity scan of the COR2 as the dot-dash curve, which is below the Saito et al. (1977) (SPM) model of the K-corona as a dotted curve and the curve of the K- and F-coronae and stray light as
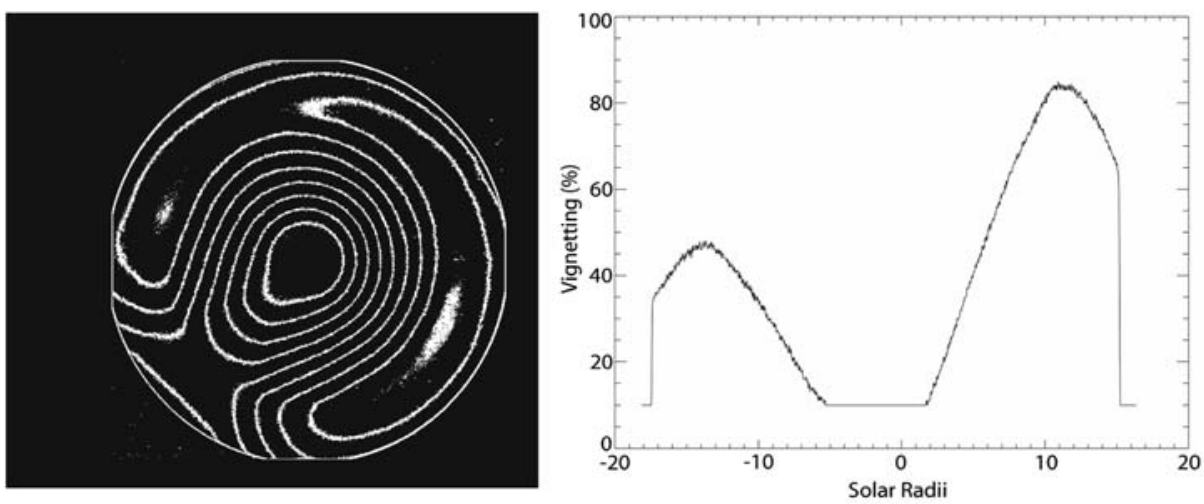

Fig. 14 Flat field response of the COR2 Ahead instrument. The plot on the right is an intensity cut diagonally from the lower left to the upper right, through the pylon 

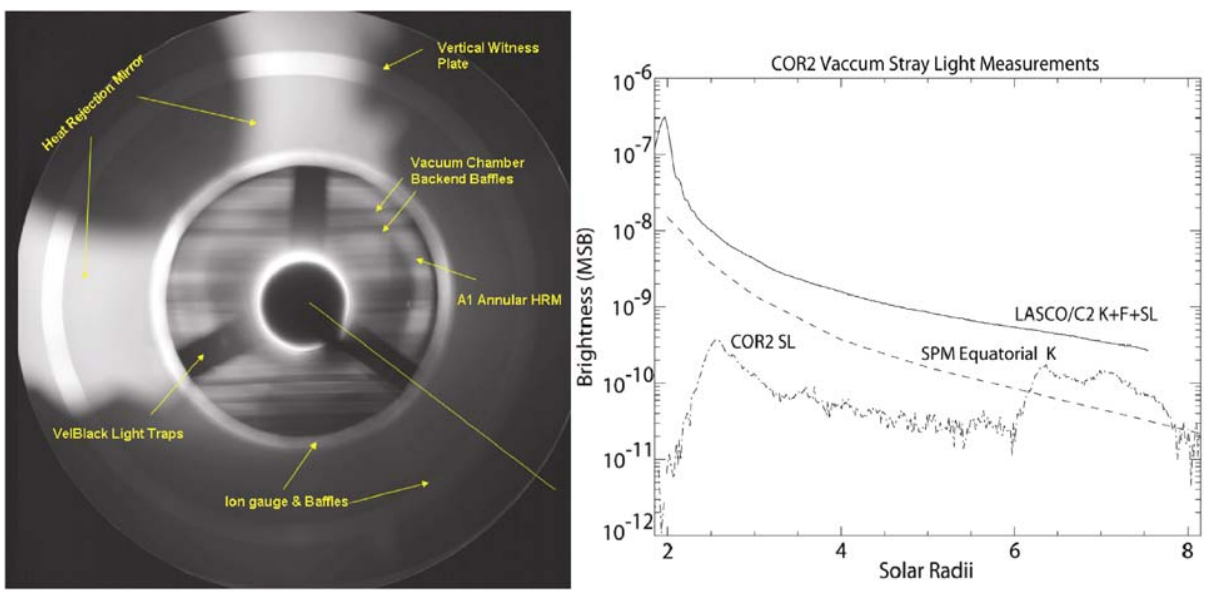

Fig. 15 Stray light performance of the COR2 Ahead instrument. The image on the left is an image taken during the stray light test. The plot on the right shows the intensity along the radial track along the line in the lower right of the image as a dot-dash. The brightness is given in units of Mean Solar Brightness

Table 5 COR2 performance requirements

\begin{tabular}{llll}
\hline Parameter & Units & Ahead & Behind \\
\hline Field of view (half-angle) & Degrees & 4 & 4 \\
Inner limit of field of view & $\mathrm{R}_{\odot}$ & 2.5 & 2.5 \\
Pixel size, full resolution & $\operatorname{arcsec}$ & 14.7 & 14.7 \\
RMS spot size (design) & $\operatorname{arcsec}$ & 15 & 15 \\
Planned exposure time & $\mathrm{sec}$ & $<4$ & $<4$ \\
Polarizer attenuation & - & $10^{-4}$ & $10^{-4}$ \\
Photometric response & $\mathrm{B} \odot / \mathrm{DN}$ & $1.35 \times 10^{-12}$ & $1.25 \times 10^{-12}$ \\
Time to complete pB sequence & $\mathrm{sec}$ & 11 & 11 \\
Image sequence cadence & $\mathrm{min}$ & 15 & 15 \\
\hline
\end{tabular}

recorded by the LASCO/C2. Note that the COR2 curve is an upper limit to the true stray light (which must be determined on-orbit) and is about one order of magnitude less than the K-coronal model.

The overall performance characteristics of the COR2 instruments are shown in Table 5.

\subsection{Operations}

The COR2 instruments will acquire only polarized images of the corona since the polarizer is always in the beam. The standard sequence will be three images at $-60^{\circ}, 0^{\circ}$, and $+60^{\circ}$, similar to COR1. All three images will be transmitted to the ground and then combined to produce total and polarized brightness images for further analysis. A low resolution, total brightness image created by the summation of the three polarized images will also be calculated onboard and transmitted through the space weather beacon channel. An alternative observation mode is to take two images at $0^{\circ}$ and $90^{\circ}$ in rapid succession without reading out the CCD in between exposures. This so-called "double" exposure corresponds to a total 
brightness image and is transmitted to the ground as a single image. This is useful way to save telemetry and increase image cadence for special observations. More details about the instrument operations are given in Sect. 11.

\section{The Heliospheric Imagers (HI)}

\subsection{Instrument Concept}

The HI objectives, like the rest of the SECCHI suite, are to make visible light observations of CMEs and other structures as they transit from the corona and into the heliosphere. The HI package consists of two small, wide-angle telescope systems (HI-1 and HI-2) mounted on the side of each STEREO spacecraft, which together view the region between the Sun and Earth. HI has no mechanisms, other than a one-shot door that protects the instrument from contamination during ground operations and launch. Thus, an image is collected in the shutterless mode, in which the intensity at each pixel is an accumulation of the static scene and a smearing of the image during readout. This smearing can be removed on the ground.

The HI instrument concept was derived from the laboratory measurements of Buffington et al. (1996) who determined the scattering rejection as functions of the number of occulters and the angle below the occulting edge. The concept is not unlike observing the night sky after the Sun has gone below the horizon.

While the specific concept used here has not been flown before, two other instruments have flown which have validated the ability to measure the electron scattered component against the strong zodiacal light and stellar background. The Zodiacal Light Photometer (Pitz et al. 1976) on the Helios spacecraft, launched in 1974, and from the Solar Mass Ejection Imager (SMEI) instrument (Eyles et al. 2003), on the Coriolis spacecraft, launched in 2003 have demonstrated that a properly baffled instrument can detect CMEs (Tappin et al. 2003).

The performance specifications for $\mathrm{HI}$ are listed in Table 6. The HI-1 and HI-2 telescopes are directed to angles of about 13 degrees and 53 degrees from the principal axis of the instrument, which in turn is tilted upwards by 0.33 degrees to ensure that the Sun is sufficiently below the baffle horizon. Thus, the two fields of view are nominally set to about

Table 6 Performance specifications of the HI telescopes

\begin{tabular}{llll}
\hline & HI-1 & HI-2 & Units \\
\hline Direction of centre of FOV & 13.98 & 53.68 & Degrees \\
Angular field of view & 20 & 70 & Degrees \\
Angular range & $3.98-23.98$ & $18.68-88.68$ & Degrees \\
Image array $(2 \times 2$ binning) & $1024 \times 1024$ & $1024 \times 1024$ & Pixels \\
Image pixel size & 70 arcsec & 4 arcmin & arcsec \\
Spectral bandpass & $630-730 \mathrm{~nm}$ & $400-1000 \mathrm{~nm}$ & nm \\
Nominal exposure time & $12-20 \mathrm{~s}$ & $60-90 \mathrm{~s}$ & sec \\
Typical exposures per image & 150 & 100 & min \\
Nominal image cadence & $60 \mathrm{~min}$ & $120 \mathrm{~min}$ & $\mathrm{~B} \odot$ \\
Brightness sensitivity & $3 \times 10^{-15}$ & $3 \times 10-16$ & $\mathrm{~B} \odot$ \\
Straylight rejection (outer edge) & $3 \times 10^{-13}$ & $10^{-14}$ & \\
\hline
\end{tabular}


Fig. 16 The geometrical layout of the HI fields of view and the major intensity contributions (based on a figure from Socker et al. 2000)

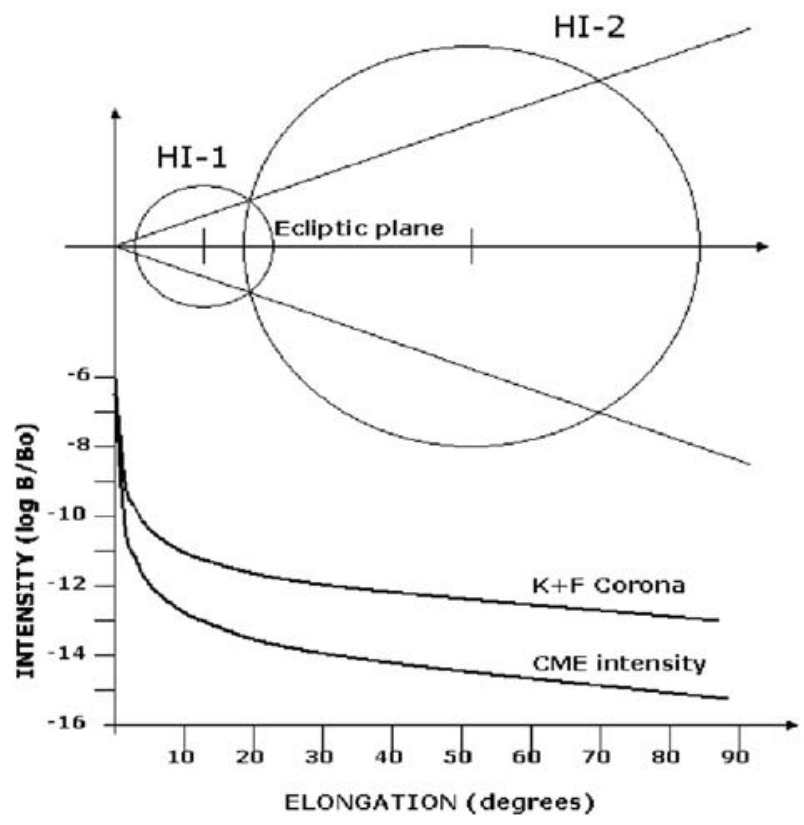

14 and 54 degrees from the Sun, along the ecliptic line, with fields of view of 20 and 70 degrees, respectively. This provides on overlap of about 5 degrees.

The geometrical layout of the fields of view of the SECCHI instruments is shown in the top half of Fig. 16. The HI-1 and HI-2 fields provide an opening angle from the solar equator at $45^{\circ}$, chosen to match the average size of a CME. This configuration provides a view of the Sun-Earth line extending from the COR2 outer limit to Earth. Since these observations are done from both spacecraft a stereographic view of an Earth-directed CME is obtained as it propagates through the inner heliosphere. In the bottom half of Fig. 16 are shown the major contributions to the observed intensities: the sum of the F-corona (zodiacal light) and $\mathrm{K}$-corona (electron scattering), and the anticipated CME intensities. Note that the $\mathrm{F}+\mathrm{K}$ coronal intensity is about two orders of magnitude brighter than the CME signal.

The anticipated instrument stray light level must be at least an order of magnitude less than the F-coronal signal which can be seen, from Fig. 16, to require levels of better than $\sim 3 \times 10^{-13} \mathrm{~B}_{\odot}$ for $\mathrm{HI}-1$ and $\sim 10^{-14} \mathrm{~B}_{\odot}$ for $\mathrm{HI}-2$, where $\mathrm{B}_{\odot}$ is the mean intensity of the solar disk. In contrast, the brightness sensitivity requirement is based on the need to extract the CME signal from the other signal sources, which demands the detection of CME intensities down to $3 \times 10^{-15} \mathrm{~B}_{\odot}$ and $3 \times 10^{-16} \mathrm{~B}_{\odot}$.

\subsection{Baffle Design}

The baffle design is the key to the HI concept. As shown in Fig. 17, the baffle sub-systems consist of a forward baffle, a perimeter baffle along the sides and rear and the internal baffle system. The forward baffle is designed to reject the solar disk intensity, reducing straylight to the required levels. The perimeter baffle is principally aimed at rejecting straylight from the spacecraft, and the internal baffle system is aimed at rejecting light from the Earth and stars.

The forward baffle protects the HI-1 and HI-2 optical systems from solar light using a knife-edge cascade system, as demonstrated in Fig. 18. The five-vane system allows the re- 

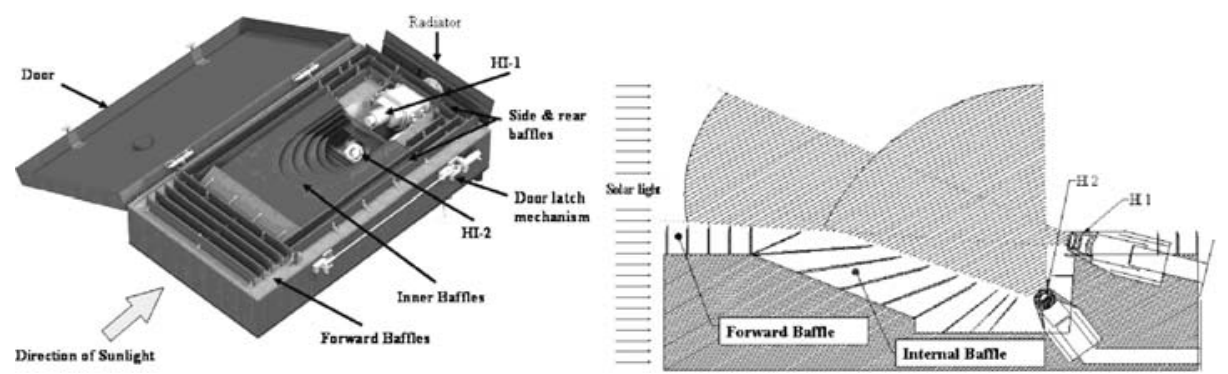

Fig. 17 Left: The Heliospheric Imager structural concept. Right: A schematic side view of the optical configuration, demonstrating the two fields of view of the two telescopes and the orientation of the interior baffles

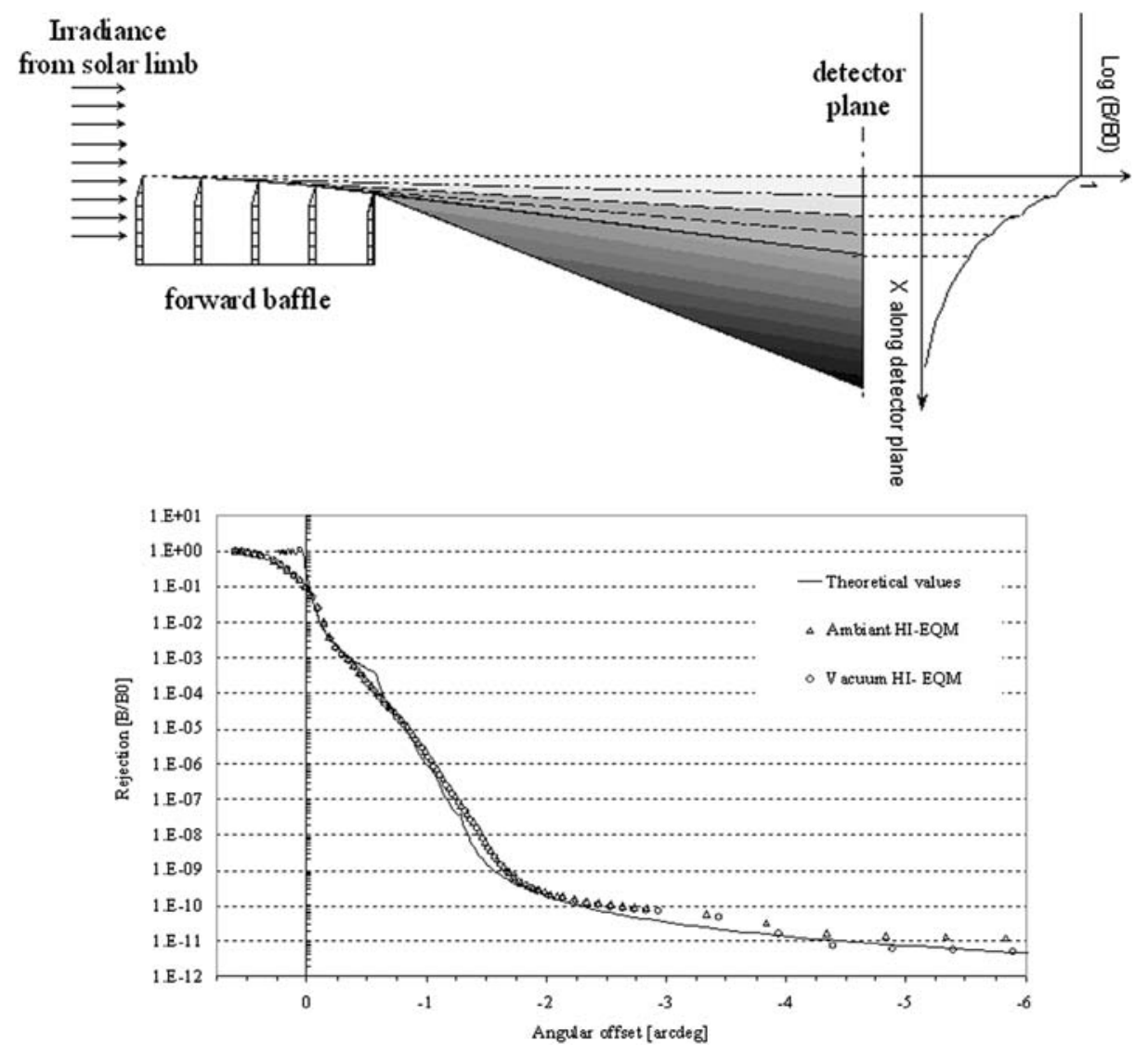

Fig. 18 Comparison of observations of the diffraction from the cascade knife-edge system of the forward baffle system with the theoretical prediction

quired rejection to be achieved, as computed using Fresnel's second order approximation of the Fresnel-Kirchhoff diffraction integral for a semi-infinite half-screen. The schematic plot in the top panel of Fig. 18 shows the nature of the function $\log \left(\mathrm{B} / \mathrm{B}_{\odot}\right)$, where $\mathrm{B}_{\odot}$ is the solar brightness, plotted with distance below the horizon. The heights and separations of 
the five vanes have been optimized to form an arc ensuring that the $(n+1)$ th vane is in the shadow of the $(n-1)$ th vane. The computed global rejection curve for this system is plotted in Fig. 18 (bottom panel), together with measurements made using a full 5-vane mock up baffle in ambient and vacuum conditions which show good adherence to the predicted rejection levels (Defise et al. 2003).

The perimeter baffle (sides and rear vane systems) protects the HI optical systems from reflection of sunlight off spacecraft elements lying below the horizon defined by the baffles, including the High Gain Antenna, door mechanisms etc. However, one spacecraft element does rise above the baffles, namely the $6 \mathrm{~m}$ long monopole antenna of the SWAVES instrument, although it is not in the field of view of the telescopes. However, from the measured reflection measurements provided by the SWAVES team and calculations, the level of reflected light from the monopole entering the entrance aperture will be insignificant.

The internal baffle system consists of layers of vanes which attenuate unwanted light from multiple reflections into the HI-1 and HI-2 optical systems, mainly from stars, planets the Earth, zodiacal light and the SWAVES monopole. Although the Earth, stars and planets may be within the HI fields, the internal baffle system limits the uniform background scattered into the optical systems.

\subsection{Optical Systems}

Figure 17 shows the locations of the HI-1 and HI- 2 optical units. The optical configurations for these are shown in Fig. 19. These systems have been designed to cater for wide-angle optics, with $20^{\circ}$ and $70^{\circ}$ diameter fields of view, respectively, with good ghost rejection, using radiation tolerant glasses (as indicated by the notation for each lens). The HI-1 lens system has a focal length of $78 \mathrm{~mm}$ and aperture of $16 \mathrm{~mm}$ and the HI-2 system has a $20 \mathrm{~mm}$ focal length and a $7 \mathrm{~mm}$ aperture. The design is optimized to minimize the RMS spot diameter and anticipates an extended thermal range from $-20^{\circ} \mathrm{C}$ to $+30^{\circ} \mathrm{C}$. The detector system at the focus in each case is a $2048 \times 2048$ pixel 13.5 micron CCD.

The lens assemblies have undergone detailed design and test procedures and one of the key requirements is on the stray light rejection; the lens systems are mounted in blackened barrels. For HI-1 and HI-2 stray light rejection is measured to be at $10^{-3}$ or lower. This combined with the stray light measurement of the front baffle, shown in Fig. 18, provides an overall light rejection level of $10^{-11}-10^{-14}$ for HI-1 and $3 \times 10^{-15}$ for HI- 2 . These values are better than the straylight requirements (Table 6).

\subsection{Mechanical Structure}

The basic design concept for $\mathrm{HI}$ can be seen in Fig. 17. The instrument is a $720(L) \times$ $420(W) \times 240(H) \mathrm{mm}$ box. A door covers the optical and baffle systems during launch and the initial cruise phase activities. The door is designed for single-use operation and will be opened after all contamination generating spacecraft propulsion events have occurred prior to insertion into heliocentric orbit.

The HI structure has 6 components: (1) a five-sided box with stiffening braces, (2) the linear baffle assembly, (3) the perimeter baffle assembly, (4) an interior baffle assembly, (5) telescope trunion mounts and (6) the FPAs. The box is an aluminum honeycomb structure with carbon-fiber (CFRP) face sheets. The face sheets were either 3 or 6 layers in which the layers were oriented at 60 degrees to each other to create pseudo-isotropic thermal characteristics. The 3 layer layups were used in non-load bearing areas. The temperature 


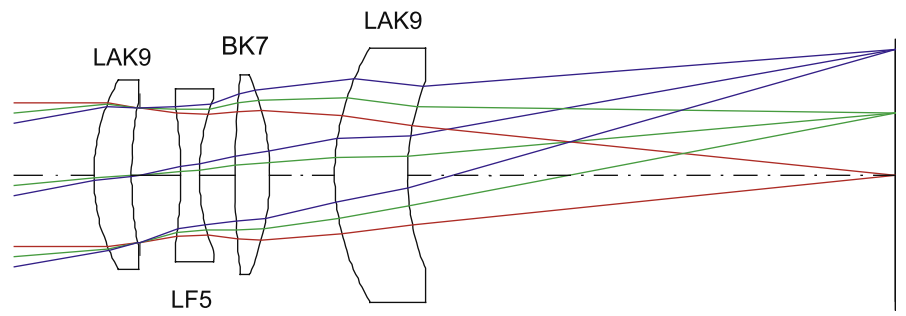

$88 \mathrm{~mm}$

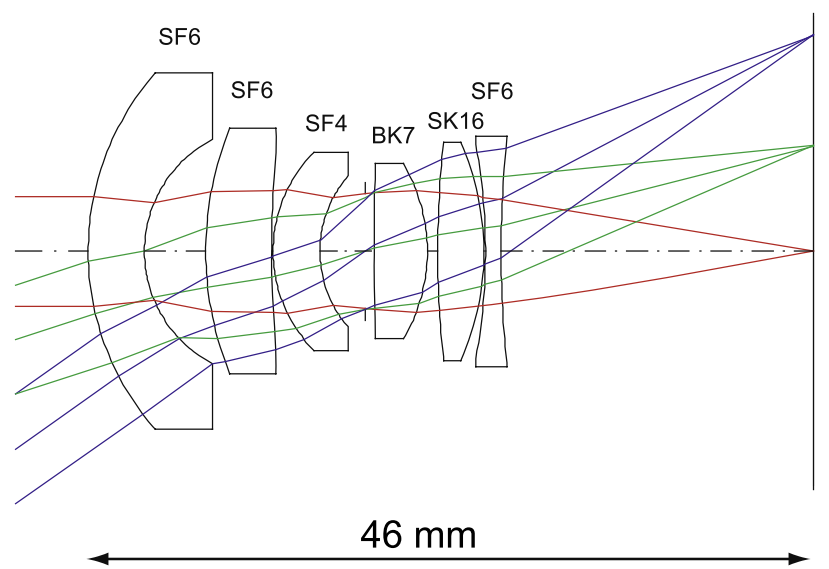

Fig. 19 The optical configurations of the HI-1 (upper) and HI-2 (lower) lens assemblies

extremes in the instrument are expected to be on the order of $100^{\circ} \mathrm{C}$ and the relationship between the front baffle assembly to the HI- 1 and HI- 2 optical axes must be held to within 125 and 240 arcsec (respectively). The baffles are made with 6-8 layers of face sheet layups. The exposed CFRP surfaces were painted with Chemglaze Z307 black paint. The structure met the requirement to have the first natural frequency resonance at or above $50 \mathrm{~Hz}$. The spacecraft mounts were similar to those described in Sect. 7.1.

\subsection{Operations}

In order to extract the CME signal from the much more intense background of Zodiacal light and stellar background, the SNR must be increased over a single exposure. This is accomplished by summing individual exposures on-board. This requires that the individual images be scrubbed for cosmic rays, prior to summing and also prior to being $2 \times 2$ pixel binned. This $2 \times 2$ binning results in angular sizes of $70 \operatorname{arcsec}(\mathrm{HI}-1)$ and $4 \operatorname{arcmin}(\mathrm{HI}-2)$ per pixel. The combination of summing 50 images and $2 \times 2$ binning results in an increase in the SNR of about $14 \times$. For each telescope, Table 6 lists a nominal exposure time range and typical number of exposures per summed image. Since there is no shutter, a smearing will occur during the readout. Software to remove this effect was applied to images taken during thermal-vacuum testing with excellent results.

A simulation of the instrument performance has been reported in Harrison et al. (2005). In that study the scene was simulated by adding the contributions from the F-corona, 
K-corona, stars, planets, and the instrumental characteristics such as point spread function, stray light, photon noise and the shutterless operation. In this background a simulated CME was launched and shown to be detectable.

\section{Guide Telescope}

The SECCHI Guide Telescope (GT) is mounted on the SCIP and serves two main purposes:

1. The GT acts as the spacecraft fine sun sensor.

2. The GT provides the error signal for the EUVI fine pointing system (FPS).

The GT images the Sun onto an occulter, which is sized to block most of the solar disk and to pass only the solar limb. The intensity of the limb is measured in 4 sections by photodiodes, placed $90^{\circ}$ apart. The concept is based on the guide telescope for the TRACE Small Explorer mission.

The optical system consists of an achromatic refractor with bandpass entrance filter and a Barlow lens (Fig. 20). A set of 4 redundant photodiodes behind an occulter measure the location of the solar limb (Fig. 21). The signals from the photodiodes are amplified and passed to the SECCHI Electronics Box. Table 7 lists key parameters of the GT. In contrast to the TRACE GT, the SECCHI GT has no provisions for off-pointing the GT relative to the science telescopes. The focal length of the GT for SECCHI is shorter than for TRACE, and is different for the Ahead and the Behind observatories. This extends the range of apparent solar diameters that the GT can accommodate, and allows the GT to operate throughout the elliptical orbit of each STEREO observatory.

The analog photodiode signals from the GT pre-amplifier are sampled every $4 \mathrm{~ms}$ by a 12-bit analog-to-digital converter in the SECCHI Electronics Box. The SECCHI flight software converts the four diode voltages into pitch and yaw pointing error signals, and a set of four sun presence flags (one flag for each photodiode). Those signals are sent to the spacecraft. If all four photodiodes are illuminated, then the sun is within the linear range of the GT, all four sun presence flags are set, and the error signals are valid. If only one, two, or three photodiodes are illuminated, then the sun is within the acquisition range of the GT, and the STEREO spacecraft uses the sun presence flags to acquire the sun. The SECCHI flight software further processes the GT error signals to generate the drive voltages for the active secondary mirror of the EUVI Fine Pointing System.

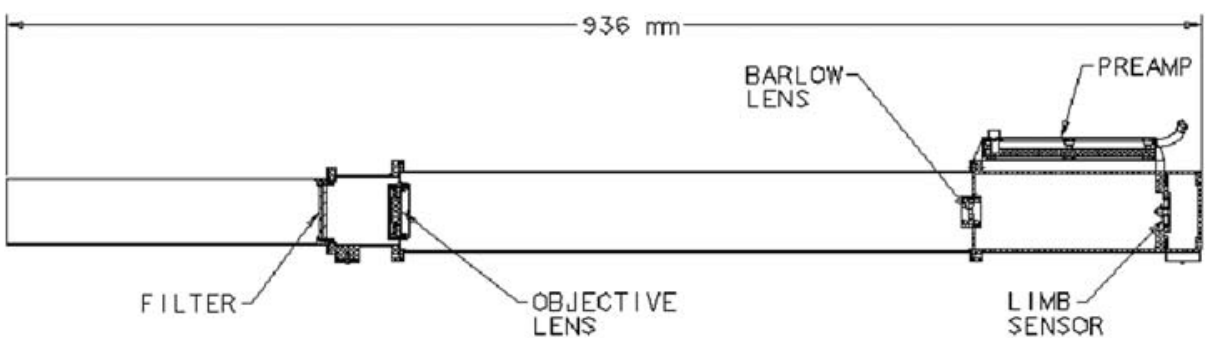

Fig. 20 Schematic cross section of the SECCHI Guide Telescope 


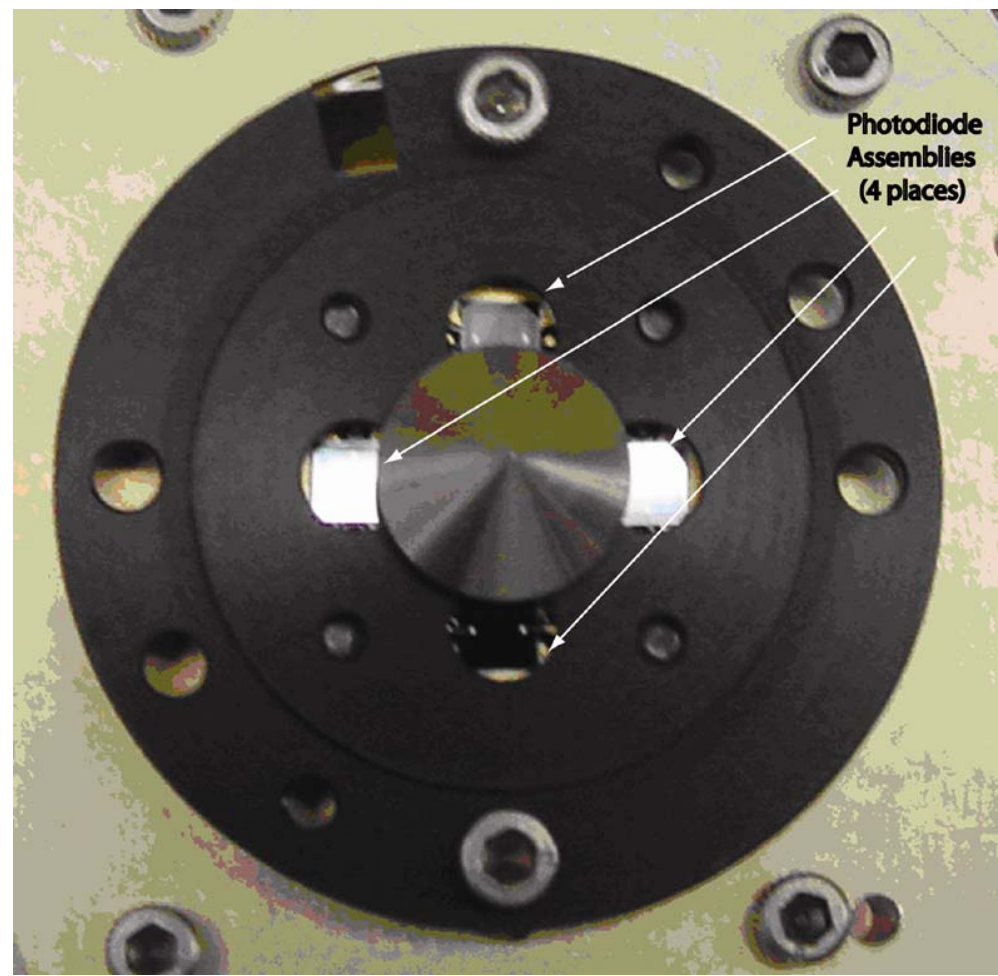

Fig. 21 Guide Telescope sensor head with occulter and photodiodes

Table 7 Key Guide Telescope parameters

Optics

Lens materials

Aperture

Focal length

Bandpass filter

Limb sensor

Telescope structure

Acquisition range

Linear range

Pre-amplifier

Noise

Sample frequency
Achromatic refractor with Barlow lens

Radiation hardened glass

$27 \mathrm{~mm}$ (aperture stop at objective lens)

Ahead: $1454 \mathrm{~mm}$, Behind: $1562 \mathrm{~mm}$

Center wavelength: $570 \mathrm{~nm}$, bandwidth: $50 \mathrm{~nm}$ (FWHM)

4 (+4 redundant) photodiodes

Aluminum

$> \pm 20 \operatorname{arcmin}$ (worst case)

$> \pm 70 \operatorname{arcsec}$ (worst case Aphelion)

Two selectable gains: sun/stimulus telescope (artificial sun) $<0.4 \operatorname{arcsec}(3 \sigma$, single sample)

$250 \mathrm{~Hz}$ (digital sampling in SEB)

\section{SCIP Bench}

The SCIP (Sun Centered Instrument Package) structure supports the four SCIP telescopes (COR1, COR2, EUVI, and GT), the two SCIP electronics boxes (SCIP CEB and MEB), and the associated harnesses and thermal blankets. The design of the SCIP structure is driven by several requirements. The structure must (1) provide easy mounting and accessibility 
for component and spacecraft integration, (2) preserve the unobstructed field of view requirements of each telescope, (3) facilitate co-alignment activities and preserve telescope co-alignment on-orbit, (4) be sufficiently stiff to avoid coupling with spacecraft and launch vehicle modes, and (5) meet a stringent mass budget while surviving test and launch loads.

The SCIP structure is a simple optical bench. The four telescopes are mounted on one side of the bench while the electronics boxes are mounted on the opposite side. Several holes through the bench allow harnessing to pass between the telescopes and the electronics. The SCIP bench is a $6.3 \mathrm{~cm}$ thick aluminum honeycomb panel $(112 \mathrm{~cm} \times 70 \mathrm{~cm})$ with high modulus graphite/cyanate ester face sheets. Face sheet layup is biased for high shear stiffness and a resulting higher torsional stiffness of the optical bench. The design provides sufficient stiffness to meet the $50 \mathrm{~Hz}$ SCIP assembly first natural frequency requirement (measured a $54 \mathrm{~Hz}$ first mode, bench torsion, during assembly vibration testing).

An additional thermal tent structure is attached to the bench to support an enclosure of thermal blankets over the telescopes. The thermal tent structure is comprised of $0.5 \mathrm{~cm}$ honeycomb panels at the sun and anti-sun ends of the bench with tubular stringers connecting the panels. Thermal blankets are draped over the structure to control the thermal and contamination environments inside the enclosure and provide insulation from the spacecraft cylinder. This generates a very uniform thermal environment for the SCIP telescopes.

\subsection{SCIP Flexure Mounts}

A key requirement of the SCIP structure is its ability to facilitate instrument co-alignment and maintain it throughout the test program and instrument life on-orbit. The key to meeting this challenging requirement is the development of a flexure-based near-kinematic mounting scheme. The scheme incorporates a set of three flexure mounts for each telescope. Each mount is very stiff in the normal and lateral directions and much less stiff in the axial direction and in the three directions of rotation. Acting as a set of three, the mounts provide a fully constrained, but not over-constrained, mounting scheme. The design of the flexure mounts has several distinct advantages: (1) The ratio of modulus in the "stiff" directions to the modulus in the "released" degrees of freedom provides an essentially kinematic mounting system. (2) The kinematic nature of the mounts minimizes thermal stress in the telescopes, preserves co-alignment on-orbit and allows shimming of the telescopes without inducing stress in the telescope tube structure. Shimming of the telescopes was performed with 5 arcsec resolution. (3) The mounts utilize snug fitting pins at each interface to prevent shifts after integration and assure co-alignment repeatability when a telescope is removed and reinstalled. (4) The titanium alloy provides high strength and stiffness with low mass, and large thermal isolation.

\subsection{Analysis and Testing}

The primary analysis tool for the SCIP structure was a MSC/NASTRAN finite element model. The SCIP optical bench was modeled in detail, including models of each telescope and of the electronics box, with a 189,000 degree-of-freedom model. Due to the developmental effort on the flexure mounts, a highly detailed (140,000 degree-of-freedom) model was constructed to analyze high stress areas and predict mount stiffness. Based on the detailed model, a simplified model of the mount was constructed for use in the assembly-level model. The full assembly-level model was used to predict assembly first frequency, assess stress levels in the structure, derive component test spectrums and interface loads, and predict on-orbit telescope alignment shifts due to thermal loads. 
Significant testing was performed to correlate the finite element results and load test the flight structures. The flexure mounts went through two rounds of prototype testing before the flight design was finalized. A monolithic telescope simulator facilitated testing of the mount system to determine its mounting stiffness. After the flight hardware was manufactured, other simulators were constructed to be used in static and dynamic load testing of the flight article. The test results were used to validate the component test spectra that were used to qualify the telescopes before they were integrated with the flight optical bench.

\section{Mechanisms}

The SECCHI SCIP telescope suite contains a total of 10 mechanisms of 6 distinct designs. EUVI has a focal plane shutter, filter wheel, and a quadrant selector. The COR1 and COR2 telescopes each have a focal plane shutter and polarizer mechanism. All three of the SCIP telescopes have a door mechanism. The HI telescopes have one mechanism, a door, which was discussed in Sect. 4.

All mechanisms have heritage from previous flight programs. The SCIP door mechanisms are based on a SOHO-LASCO design. The EUVI quadrant selector is an evolution from the mechanism used in the TRACE quadrant selector, in that there are no restrictions in the frequency of changes between telescope quadrants. The EUVI filter wheel, COR2 and COR2 polarizer wheels and shutter mechanisms are nearly identical to the ones used on GOES SXI-N (Stern 2003).

\subsection{SCIP Aperture Doors}

The re-closable doors of the EUVI, COR1 and COR2 telescopes are referred to as the SESAMEs (SECCHI Experiment Sun Aperture Mechanisms). In total there are two sets of SESAMEs, one for each SCIP assembly (Fig. 22). The prime objectives of the re-closable doors are to protect the sensitive optics of the telescopes from damage due to contamination during ground operations, but also at critical times during flight operations. The launch of

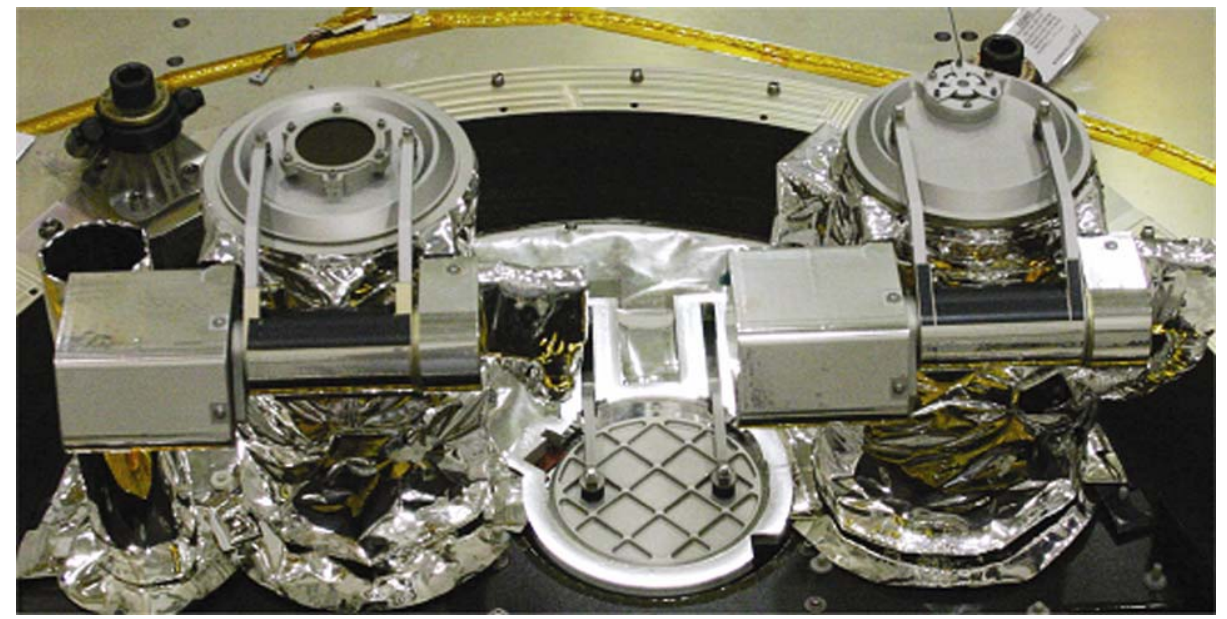

Fig. 22 Top front view onto the SCIP-B assembly after integration into observatory B. From left to right: COR 1, EUVI and COR 2 doors. The SCIP-A doors are identical 
the Delta rocket generates large acoustic vibrations requiring the door lids to be pressed tightly against the telescope entrance apertures without generating particles. The EUVI is especially sensitive because the lid has to protect the thin optical filters lying directly behind it. The doors also help to maintain the cleanliness of the optical systems from external contaminants that may be caused during thruster firings to control the orbit of the STEREO spacecraft. Because of momentum desaturation the thrusters will be fired about every 6 days on average, implying that the COR doors will be cycled about 120 times during the two-year nominal operations period. Re-closable doors are also required because the optical systems must open and close during ground testing and calibration of the optical systems.

All three doors have a similar baseline design. Each is operated by a stepper motor which drives a special screw-ball bearing mechanism to open and close the lids. In case of the malfunction of the motor a paraffin actuator acts as the failsafe device to open the door permanently. The command to "fire" the paraffin actuation is controlled by the spacecraft. The doors can be driven by the motors into different positions: superclosed, closed, cracked and open. The superclosed position presses the door lid against the aperture to withstand the launch environment, which could cause the lid to vibrate against the telescope tube. This vibration would create particles. The closed position is the normal closed position without the launch pre-load. The cracked position is an intermediate position between closed and open to permit outgassing to occur after launch. The open position is the normal position for science operations. The lid designs of EUVI, COR1 and COR2 have different sizes and structures, with calibration windows being integrated into the COR lids. The opening angle of the EUVI door is 90 degrees, that of the COR doors is 270 degrees.

\subsection{Shutters}

The EUVI, COR1 and COR2 shutter designs are based on shutters that have successfully flown on SOHO/MDI, TRACE (Akin et al. 1993), and Solar-B/FPP and will be flown on GOES-N/SXI. Both consist of a brushless DC motor with an optical encoder to which is attached a thin circular blade with a $120^{\circ}$ opening that is positioned in front of the telescope's focal plane. The motor rotates the blade to open the shutter, exposing the CCD. The blade is rotated in the same direction to close the shutter. The SECCHI shutters are capable of exposures from $40 \mathrm{~ms}$ to 67 seconds in a programmable mode that are repeatable to better than $15 \mu$ s and uniform across the CCD to $50 \mu \mathrm{s}$. The exposure length can be indefinite when open and close motions are controlled directly by the SEB.

\subsection{Quadrant Selector}

The EUVI quadrant selector is nearly identical in design to the coronagraph shutters. The motor rotates a blade with a $90^{\circ}$ opening just behind the telescope's thin-film entrance filters. The mechanism is commanded to rotate in $90^{\circ}$ increments in approximately $45 \mathrm{~ms}$ to mask off all but one of the telescope's four EUV channels.

\subsection{EUVI Filter Wheel}

EUVI also incorporates a four position filter wheel just behind the telescope's shutter. The filter wheel consists of a $101 \mathrm{~mm}$ diameter brushless DC motor surrounding a rotor with four $32 \mathrm{~mm}$ diameter through holes. The mechanism will be launched with the filter wheel in the open position, leaving the telescope beam-path open and protecting the three-filter position within the mechanism housing. Operation time between two adjacent filter positions is approximately $300 \mathrm{~ms}$. Like the SECCHI shutters, the filter wheel has heritage from TRACE, MDI, SXI, and FPP. 


\subsection{Polarizer Wheel}

The coronagraph polarizer mechanism, also known as the hollow-core motor (HCM), is the most unique of the SECCHI mechanisms. Its design is derived from a similar mechanism used with success in MDI. It has a 144 step brushless DC motor similar to that used for the EUVI filter wheel. A large-diameter, thin-section bearing supports a stainless steel rotor with Teflon toroid separators. The space inside the bearing is left open, providing a $48 \mathrm{~mm}$ clear aperture for the coronagraph light bundle. With the 144 step motor design, the polarizing optic that is mounted to the rotor can be positioned in $2.5^{\circ}$ increments. During normal observing operations, the polarizer mechanism will rotate $120^{\circ}$ with an angular repeatability of better than 30 arcseconds. It makes this move in approximately $400 \mathrm{~ms}$.

\subsection{Lifetime Requirements}

The lifetime requirements for each of the SECCHI mechanisms are 2.1 million operations for the EUVI quadrant selector, filter wheel, and shutter and 0.6 million operations for the COR polarizer mechanisms and shutters. Comprehensive life testing was completed to prove the designs of the EUVI quadrant selector, coronagraph shutter, and HCM. These mechanisms underwent vibration to levels greater that for flight, thermal functional testing, and flight like operation in vacuum to three times their design lifetime. The quadrant selector was qualified to 8 million operations, the coronagraph shutter qualified to 2.8 million operations, and the polarizer mechanism qualified to 3.5 million operations with no degradation in performance. The designs for the EUVI filter wheel and shutter were previously proven to be capable of more than 32 million operations.

\section{SECCHI Electronics}

SECCHI contains three types of electronics arranged into 4 different boxes. The main electronics is contained within the SECCHI electronics box (SEB) and is described in Sect. 9.1. The SCIP mechanisms are driven from a remote electronics box, described in Sect. 9.2. The SCIP and HI cameras are driven from remote electronics boxes, called the Camera Electronics Box (CEB) and described in Sect. 10.3.

\subsection{The SECCHI Electronics Box (SEB)}

The SEB (SECCHI Electronics Box) is the payload controller for the SECCHI Instrument Package on the STEREO spacecraft, controlling and monitoring all SECCHI hardware. A block diagram of the SEB is shown in Fig. 23. The SEB provides the instrument's interface to the spacecraft via the MIL-STD-1553 and power interfaces. It receives and distributes commands from the spacecraft and collects and converts state of health telemetry data before providing it to the spacecraft. Image data from the telescopes ( 2 channels, $100 \mathrm{MB} / \mathrm{s}$ ) is received over two SpaceWire ports and is then processed, compressed, and transmitted across the 1553 interface to the spacecraft for downlink. The SEB had to be designed to meet stringent program EMI requirements, as will be discussed briefly below. An Engineering Development Model (EDM) was designed and built before the flight hardware as a pathfinder for the designs. This EDM is now part of the software testbed that will be maintained during the life of the mission. The flight hardware underwent EMI/EMC, vibration and thermal-vacuum testing before integration with the SECCHI instrument packages. 


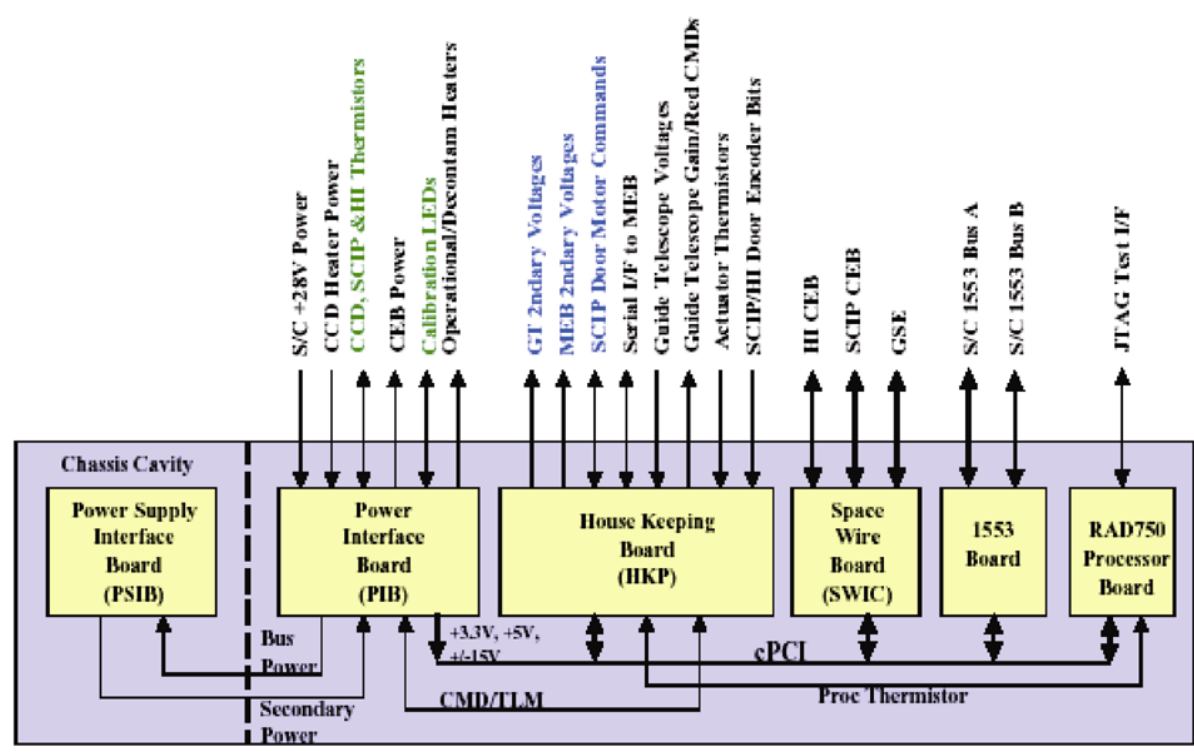

* SEB is single string

Items originate on PIB and go out through HKP Items come in through PIB \& get routed to HKP

Fig. 23 SECCHI Electronics Box (SEB) functional block diagram

\subsubsection{Motherboard}

The SEB Motherboard provides electrical connectivity between all cards housed within the SEB enclosure. It is a customized, cPCI compliant, five (5) slot, 6U back plane. The first four (4) cPCI slots which utilize the PCI signaling strictly adhere to the cPCI specification. The fifth slot which does not use the PCI signaling has been customized for communications on the user defined connectors, P3/P4/P5. Unused cPCI connectors were eliminated from the design.

\subsubsection{Electrical Power}

The SEB must interface to the spacecraft's unregulated power bus. The electrical power system provides this interface as well as power conversion, control and distribution. Due to EMI requirements, the electrical power system was divided into two (2) functional assembliesthe DC/DC converters and related circuitry (PSIB) and the power interface and distribution functions (PIB).

The PIB interfaces to the primary spacecraft power bus through a front panel connector and routes this power off the motherboard and through the EMI cavity line filter panel, to the DC/DC converters in the small EMI cavity. Regulated secondary power is then routed back from the DC/DC converters to be distributed throughout the SEB and SECCHI system by the PIB card. Internal secondary SEB power is distributed across the motherboard as required. External SECCHI power is routed through PIB I/O connectors and, in some cases, across the motherboard and out through the HKP I/O connectors due to front panel limitations. Power control signals are generated from gate arrays resident on the HKP card, 
as commanded by the RAD750 processor card. The HKP card determines the proper commands to send to the PIB and uses a serial interface across the motherboard to transfer the information. The PIB also provides motor drive power from control signals generated on the HKP. Voltage and current monitoring circuits on board provide state of health telemetry to the HKP for conversion and, ultimately, downlinking. This card also houses the operational heater drive circuits with appropriate switching for thermal control of the instrument package. The heaters are again controlled via the HKP card since the PIB has no interfaces with the processor for real estate and design simplification reasons. A second power bus from the spacecraft is distributed to SECCHI decontamination heaters through latching relays on this card.

The PSIB assembly resides in the small EMI cavity and includes three (3) DC/DC converters that provide all secondary power for the SEB and SECCHI system, as well as all necessary filtering and protection. A single converter provides $+3.3 \mathrm{Vdc}$ power for internal logic. A triple output converter provides +5 VDC for internal and external legacy logic and $+/-15 \mathrm{Vdc}$ power for analog functions. The third converter provides $+15 \mathrm{Vdc}$ dedicated for "noisy" motors and mechanisms. Primary and secondary power interfaces, to the main cavity, are through an EMI filter panel.

\subsubsection{RAD750 Processor}

The SEB RAD750 processor, procured from BAE Systems, provides all processing required for the SECCHI system and the control of all SEB circuitry via the cPCI backplane. At the core is a custom Power PCI Bridge chip providing the interface between the PowerPC 750 and the cPCI bus. The primary computing resource is the radiation hardened PowerPC 750. The RAD750 card provides for 256 kbytes of EEPROM StartUp ROM (SUROM) and 128 Mbytes of SDRAM. SDRAM EDAC can correct nibble errors and detect double nibble errors. The card provides a self-refresh mode and scrubbing for the SDRAM. The RAD750 is capable of 120MIPS and is being run at $116 \mathrm{MHz}$ in the SECCHI application. This card is the only $3 \mathrm{U}$ card in the SEB.

\subsubsection{MIL-STD-1553 Interface Card (1553)}

The 1553 card provides a fully compliant MIL-STD-1553 Remote Terminal (RT) bus interface to the spacecraft and 3 Mbytes of EEPROM storage for software application code. The 1553 interface is the only communications interface with the host spacecraft. All commands, data uploads and telemetry are communicated across this bus. Full redundancy makes this a very robust interface. The EEPROM is arranged as two (2) banks of 1.5 Mbytes $\times 16$ bits wide. This storage is fully accessible across the $\mathrm{cPCI}$ bus and provides for redundant flight software storage.

\subsubsection{SpaceWire Interface Card (SWIC)}

The SWIC provides two (2) $100 \mathrm{Mb} / \mathrm{s}$, simultaneous SpaceWire compatible links to the SCIP and HI instruments respectively. All image data and camera setup commands are transferred using these interfaces. The TSS901E ASIC, from Atmel, provides these SpaceWire links. 256 Mbytes of SDRAM allows for temporary image storage while awaiting retrieval and processing by the RAD750, and can also be used by the processor for data storage or program execution. The SDRAM is complete with programmable refresh, scrubbing and EDAC. To efficiently move large amounts data, the PCI interface can be configured for DMA transfers, on to or off of the SWIC. 


\subsubsection{Housekeeping Card (HKP)}

The HKP is the bridge between the analog interfaces and the digital cPCI bus for the SEB. It controls serial data transfers with the SCIP mechanisms controller (Mechanisms Electronics Box-MEB hardware) for shutter control and other commands. All SECCHI door status is monitored and made available by the HKP. A regulated current source provides selectable drive for SECCHI calibration LED illumination. Analog Guide Telescope signals are received and digitized by the $\mathrm{HKP}$ at a $250 \mathrm{~Hz}$ rate for delivery to the processor for transfer to the spacecraft's Attitude Control System (ACS). Sixty three analog telemetry points, including voltage, current and temperature sensors, for both SEB internal and SECCHI external, are digitized ( 0 to $+10 \mathrm{~V}$ range, 12 bit conversions) and made available to the system processor for use in fault detection and isolation and general state of health monitoring.

\subsubsection{Enclosure}

The enclosure was machined from a single block of aluminum. It includes 2 compartments allowing EMI isolation of the internal DC/DC converters. The bottom and one side have removable covers with labyrinth seals. Individual cards are installed from the top, with each card having a connector bracket that provides additional EMI protection and structural tie points. One final top cover, with clearance for each connector, completes the EMI seal. The DC/DC converters reside in a separate "EMI cavity" isolated from the main cavity. The interface between the 2 cavities is achieved with an EMI-tight, filter capacitor panel. The overall dimensions are $198 \times 292 \times 267 \mathrm{~mm}(H \times W \times L)$, weighing $9.4 \mathrm{~kg}$.

\subsection{Mechanism Electronics Box (MEB)}

All of the SECCHI SCIP mechanisms, with the exception of the telescope doors, are controlled by the SECCHI Mechanisms Electronics Box (MEB), which is mounted on the underside of the SCIP optical bench. Physically, the MEB is a $210 \times 56 \times 171 \mathrm{~mm}$ box with a mass of approximately $1.4 \mathrm{~kg}$ with connectors dedicated to power and commands from the SEB and each of the telescopes. The MEB inputs are a Low-Voltage Differential Signal (LVDS) from the SECCHI SEB, $5 \mathrm{~V}$ power for the MEB and mechanism encoders, $\pm 15 \mathrm{~V}$ power to drive mechanism motors. The three LVDS inputs to the MEB from the SEB include a strobe, clock signal, and a serial command signal. A single return LVDS signal is sent back to the SEB. The MEB contains two boards and each has two Field Programmable Gate Arrays (FPGAs). The FPGAs on the first board control the EUVI shutter, quadrant selector, and filterwheel, and drive the EUVI Fine Pointing System (FPS). The two FPGAs on the second board each control a shutter and polarizer mechanism on each of the coronagraphs.

The MEB accepts low level commands such as "load target position," "move clockwise" to that position, and "read current encoder position." These commands are sequenced by the SEB and there is no command buffer in the MEB. Table 8 provides a partial listing of commands accepted by the MEB in the order in which they are typically employed. MEB commands are 16-bit and consist of an 8-bit command word and an 8-bit variable word such as exposure time for a shutter or target position for the HCM.

The mechanism motors are driven by a $15 \mathrm{~V}$, three-phase signal generated by the MEB in response to the signal provided by the optical encoders in each of the SECCHI mechanisms. The SECCHI MEB also drives the EUVI FPS, the design of which is discussed in a separate section. The MEB accepts commands from the SECCHI SEB to derive three analog voltages from $0-10 \mathrm{~V}$ to drive the ISS PZTs. There is no PZT or mirror position feedback to the MEB. 
Table 8 MEB commands

\begin{tabular}{|c|c|}
\hline Command & Action \\
\hline RESET & $\begin{array}{l}\text { - HCM and filter wheel target and encoder reading cleared } \\
\text { - Shutters rotate to "ready for exposure" position, exposure time cleared } \\
\text { - Quadrant selector rotates to } 284 \AA \text { Auadrant, target cleared }\end{array}$ \\
\hline LOAD TARGET & HCM, filter wheel, and quadrant selector desired position saved \\
\hline MOVE CW & Mechanism moves to saved target \\
\hline LOAD & Shutter desired exposure time saved \\
\hline EXPOSURE & \\
\hline CW EXPOSURE & $\begin{array}{l}\text { Shutter makes a } 120^{\circ} \text { clockwise move to open position, waits for desired saved expo- } \\
\text { sure time, and makes a } 240^{\circ} \text { clockwise move to closed/ready position }\end{array}$ \\
\hline READ ENCODER & MEB returns to the SEB the current position of the mechanism \\
\hline READ STATUS & MEB returns to the SEB the current status of the mechanism \\
\hline
\end{tabular}

\section{Charge Couple Device (CCD) Cameras}

\subsection{CCD Detectors}

Each of the scientific instruments on SECCHI uses a three-phase, back-illuminated, nonnon-inverted mode (to ensure good full well capacity, 150k to 200k electrons), CCD model CCD42-40 manufactured by E2V in the United Kingdom. There are $2048(H) \times 2052(V)$ image pixels, each measuring 13.5 micron on a side and providing a total imaging area of $27.6 \mathrm{~mm}$ square. The total readout is $2148 \times 2052$ pixels, providing 100 columns of nonimaging over and under-scan region for camera calibration and engineering purposes. Two readout ports on the left and right side of the imaging area are available. In principle the device may be fully read through either port, or through both ports to double the readout speed. To save mass the SECCHI instruments use only one port, which is hard wired through the harness in our flight configuration, although non-flight harnesses were developed to test from either port. The CCDs used for visible light detection (COR-1, COR-2, HI-1 and HI-2) have an anti-reflective coating on their backside (illuminated side). Quantum efficiency (QE) of these devices is roughly $80 \%$ at $500 \mathrm{~nm}, 88 \%$ at $650 \mathrm{~nm}, 64 \%$ at $800 \mathrm{~nm}$, and $34 \%$ at $900 \mathrm{~nm}$. The backside of the EUVI CCD has no coating in order to provide sensitivity at shorter wavelengths, with a quantum efficiency of $74 \%$ at $17.1 \mathrm{~nm}, 70 \%$ at $30.3 \mathrm{~nm}$, and $46 \%$ at $58.4 \mathrm{~nm}$. CCDs are operated at a temperature design point of $<-65^{\circ} \mathrm{C}$.

The CCD is mounted on a custom ceramic carrier, which is precision mounted on an invar chip carrier. Electrical connections are made through a flex-print cable secured to the carrier, and a 25-pin micro-D connector. Figure 24 shows the SECCHI CCD in its flight package. The CCDs have excellent vertical and horizontal charge transfer efficienciesbetter than $99.999 \%$. The dark current generation rate is better than $2 \mathrm{nA} / \mathrm{cm}^{2} / \mathrm{s}$ at $20^{\circ} \mathrm{C}$, which is equivalent to 24,000 electrons per pixel/s.

\subsection{Focal Plane Assembly (FPA)}

The SECCHI Focal Plane Assembly (FPA) provides the SECCHI CCDs with physical mounting, optical positioning, electrical connection, and thermal cooling. The basic FPA 


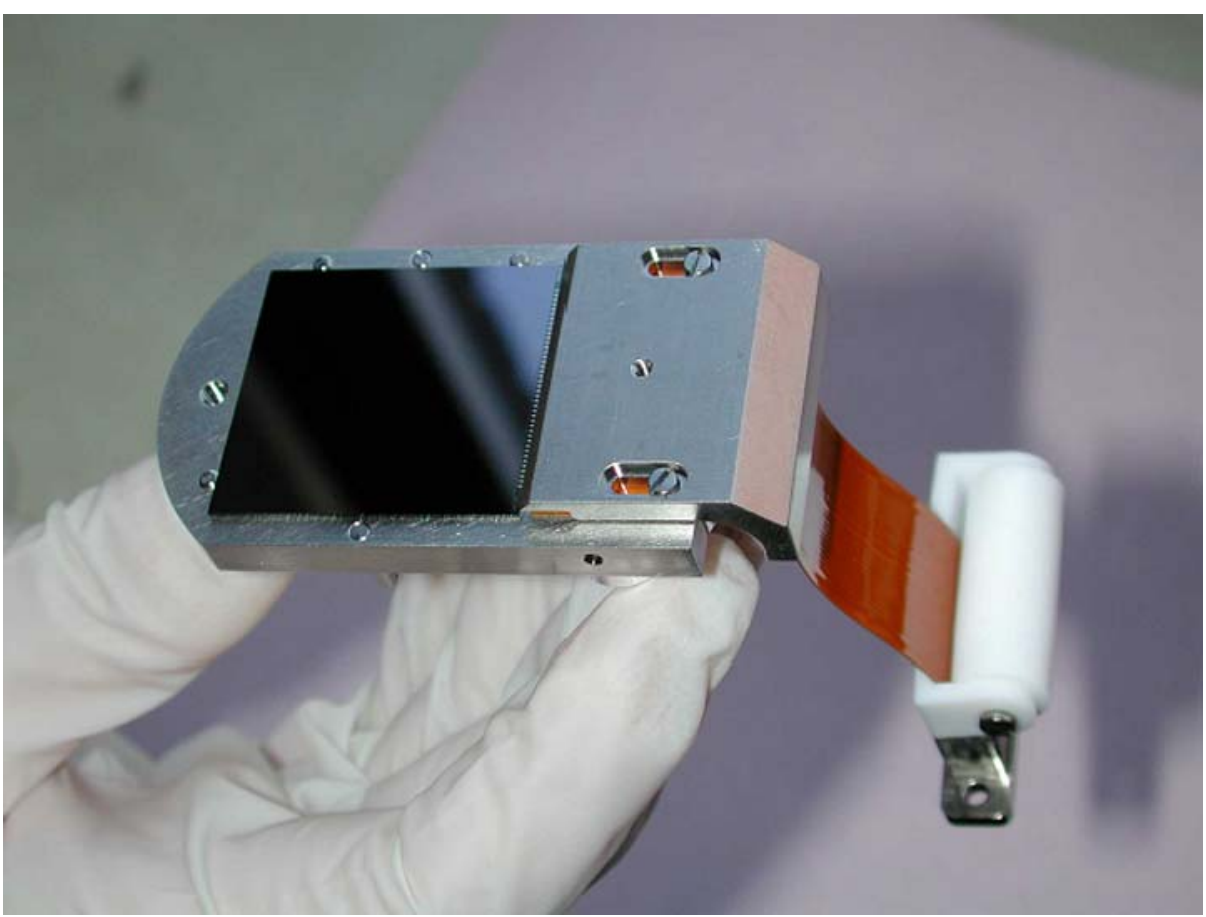

Fig. 24 SECCHI CCD in its package with flexprint harness attached. The CCD size is $28.1 \mathrm{~mm} \times 30.3 \mathrm{~mm}$

design for SECCHI was used on all five telescopes. All five assemblies are nearly identical except for differences in the length and shape of the cold finger, radiator size and shape and focal plane masks.

The requirements for the design of the FPA were: (1) Cool the CCD using passive radiation techniques to $<-65^{\circ} \mathrm{C}$ using an aluminum cold finger coupled to a surface radiating to deep space; (2) Position the CCD at the focal plane of the telescope, perpendicular to the optical axis and aligned to the optical axis; (3) Keep the CCD positioned at room and operating temperatures; (4) Provide a capability to add a spacer in between the FPA and telescope mounting flange to account for different focal plane positions of the various telescopes and to account for any focal plane shift between room and operating temperatures; (5) Provide a capability to heat the CCD to at least $30^{\circ} \mathrm{C}$ to drive off contaminants and to "anneal" the CCD after radiation damage; (6) Provide a cold cup at a temperature slightly colder than the CCD to act as a contamination shield for the CCD.

Figure 25 shows a cutaway of an FPA assembly as viewed from the front/side and also in the lower right as viewed from the rear/side. At operating temperatures, the CCD will be below $-60^{\circ} \mathrm{C}$ and the mounting flange will be about $20^{\circ} \mathrm{C}$. The $\mathrm{CCD}$ is cooled to this low temperature by conducting away the heat from the CCD through an aluminum "cold finger" which then is attached at the back end to an aluminum thermal radiator plate that has a clear view to deep space. The CCD chip carrier is first bolted onto an invar block which has a heater coil in between the carrier and the block. The heater coil can heat the CCDs to above $50^{\circ} \mathrm{C}$. The invar block is attached to the cold finger, which runs inside an outer support tube and attaches to the thermal radiator. The interior of the outer support tube and the cold finger are gold plated to reduce the heat transmission between the two. At the mechanical interfaces 


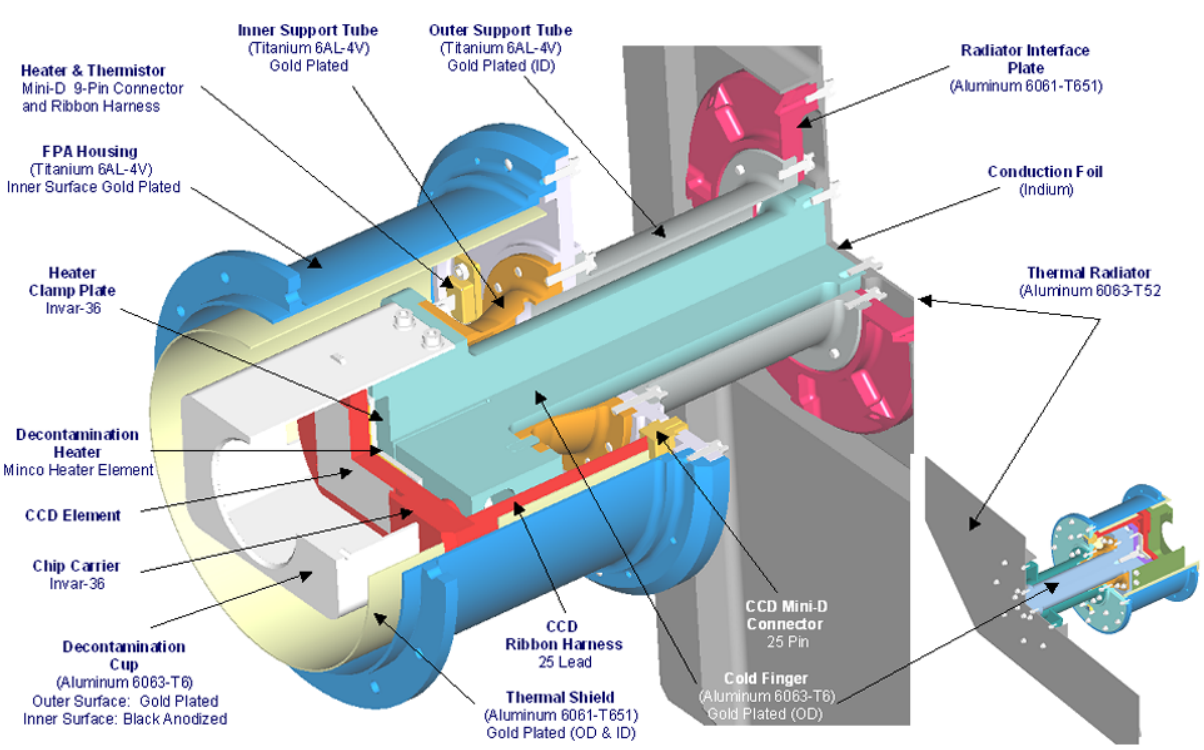

Fig. 25 Focal plane assembly

on either end of the cold finger an indium foil is sandwiched in the joint to minimize the heat conduction loss across the joint. A titanium inner support tube is attached to the cold finger on one end and the FPA housing, made of titanium. Using titanium minimizes heat conduction from the instrument to the cold finger. The front of the FPA housing attaches to the rear of the telescope housing. Attached directly to the cold finger is a cylinder that surrounds the CCD and is colder than the CCD since it has fewer mechanical interfaces and does not have the heat generated by the CCD. This cylinder (decontamination cup) collects any volatiles that are driven off the CCD during the "bake-out" process.

\subsection{Camera Electronics Box (CEB)}

The CEB must provide the appropriate control signals to each of the telescope CCDs, accept the analog signal out, digitize the signal to 14 bits and send the image data to the SEB. The CEB will collect housekeeping information to be sent to the SEB. The camera requirement calls for a CCD pixel readout rate of $1 \mathrm{Mpixels} / \mathrm{s}$, yet also dictates very challenging low mass and low power budgets. ASIC and surface-mount packaging technologies help minimize the size, mass, and power requirements of the cameras. Controlling three CCDs from the SCIP box, and two CCDs from the HI box minimizes the overhead arising from the spacecraft interfaces and the power converters, and thus the overall size, mass, and power of the camera controllers.

Figure 26 is a schematic of the CCD Camera Electronics Box (CEB). It comprises (1) Two (HI) or three (SCIP) CCD Driver Cards; one dedicated to each of the CCDs. A block diagram of the CCD Driver Card is shown in Fig. 27. (2) A Camera Interface Card that provides a common interface between the three CCD Driver Cards and the SECCHI instrument computer. (3) A DC-DC Power Converter mounted in an internal screened housing in the base of the electronics box. (4) A backplane interface for inter-connection of the daughter PCBs. 


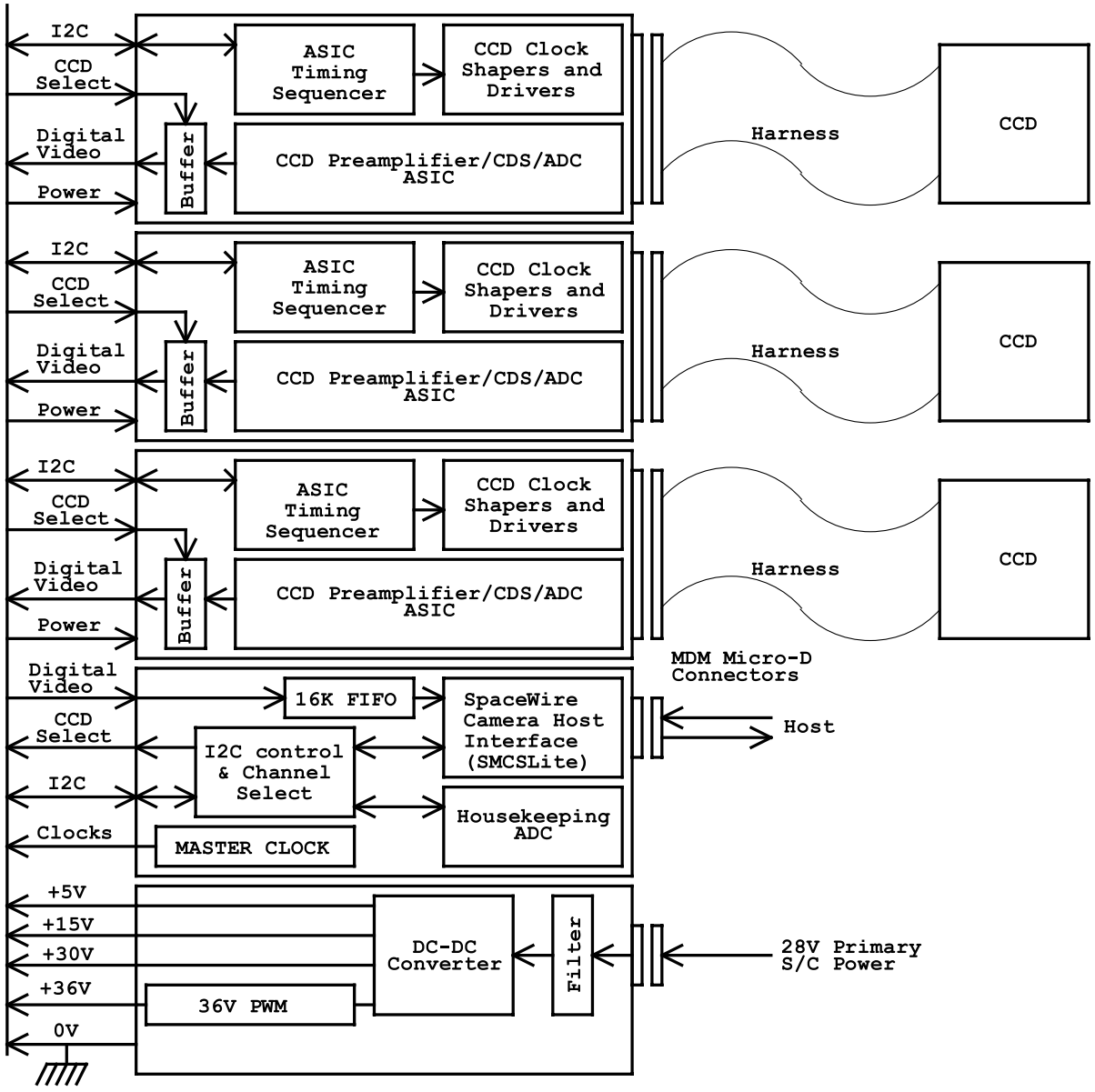

Fig. 26 CEB architecture

Each CCD Driver Card (Fig. 27) accommodates a dedicated waveform generator and sequencer ASIC, CCD clock drivers, low noise DC bias supply generators, a CCD video preamplifier, correlated double sampler (CDS), and 14 bit analog to digital converter (ADC) system.

The camera electronics exploits the same basic waveform generator ASIC and CCD clock driver circuit topologies designed for the SMEI CCD cameras (Eyles et al. 2003), but updated for faster pixel readout rate for SECCHI. Each CCD is clocked from its own dedicated sequencer and clock drivers, and is read out through its own 14 bit CDS/ADC video processor. The 14 bit CDS/ADC video processor is implemented in an ASIC which is a special purpose designed, radiation tolerant chip developed at RAL.

Each camera communicates with the SECCHI instrument computer via an IEEE1355/ SpaceWire link, enabling camera programming, camera command, gathering of housekeeping data, and the transmission of digitized CCD video data. The data transmission rate is up to $100 \mathrm{Mbits} / \mathrm{s}$, employing the SpaceWire LVDS hardware interface standard. Exposure timing for each CCD is controlled directly from the SECCHI instrument computer. Appropriate programming and control of the camera waveform generator ASICs enables updating 


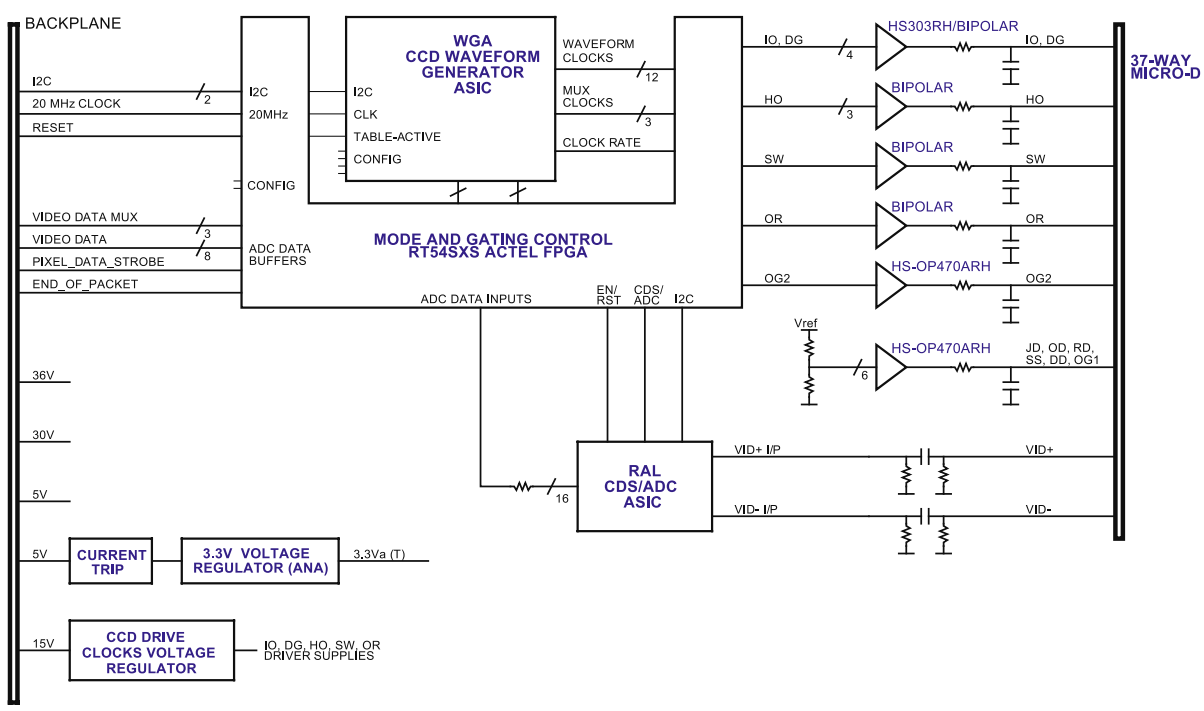

Fig. 27 CCD driver card

of CCD readout waveform patterns and readout tables, and the initiation of various readout modes e.g. clearing, exposure, full-frame, windowed, or continuous readout. Pixel binning, programmable video gain, and programmable video DC offset level are also supported.

Each camera contains a DC-DC power converter that converts the incoming $28 \mathrm{~V}$ spacecraft primary power to the required $+5 \mathrm{~V},+15 \mathrm{~V},+30 \mathrm{~V}$, and $+36 \mathrm{~V}$ secondary supplies. The camera's $28 \mathrm{~V}$ power supply input is in-rush current limited, and current-trip protected. The converter's output supply rails are also filtered to minimize any noise and/or currentswitching transitions on the supply rails to the CCD drive circuitry. The complete power converter system is screened from the CCD drive electronics within its own, shielded subhousing within the CEB.

The survival power and decontamination heater power supplies for the CCDs are not routed through the camera boxes, but go directly from the SECCHI instrument computer to the CCD heads. Similarly, the temperatures of the CCDs are monitored directly from the SECCHI instrument computer and not through the camera electronics units. Housekeeping telemetry from the camera electronics enables the controller's secondary power supply voltage rails and internal temperature to be monitored.

The SCIP camera electronics enclosure is $195 \mathrm{~mm} \times 126 \mathrm{~mm} \times 85 \mathrm{~mm}$, including all mounting feet, connector, and fixing screw protrusions. The HI camera electronics enclosure is slightly smaller $(195 \mathrm{~mm} \times 126 \mathrm{~mm} \times 70 \mathrm{~mm})$ to conserve volume and mass.

Operationally, the camera will respond to the following modes:

Clear Clearing of the CCD will take advantage of the CCD's 'dump-drain' running adjacent to the serial output register. Appropriate programming of the ASIC waveform generator will allow the entire array to be cleared any number of times prior to integration.

Integration During integration, the CCD's parallel register clocks will be held at appropriate voltage levels. The serial register clocks can be individually programmed to be high, low, or clocking. Dither clocking of the parallel register clocks will be possible to help minimize dark current generation, and to allow some degree of testing at room temperature. 
Readout Appropriate programming of the ASIC waveform generator will allow any number of readout modes including:

(1) full-frame readout of $n$ lines,

(2) windowed readout of at least two windows (dump $n$ lines, read $x$ lines, dump $m$ lines, read $y$ lines, etc.),

(3) full-frame, or windowed readout with $n \times m$ pixel binning (pixel binning parameters can be different between windows provided that the windows are not overlapping),

(4) continuous clocking.

The size of the readout area (or image format) is programmable without restrictions. Multiple windowed readout is possible up to the memory limits of the ASIC's internal 'readout table' memory. Window co-ordinates are programmable within CCD 'readout tables', but must be specified from the control computer. Any variety of camera operating cycles will be possible by appropriate programming of the waveform generator ASIC. Cycles may be implemented as a series of short 'readout tables', individually initiated from the control computer; or alternatively as one long 'readout table' initiated only at the beginning.

\section{Flight Software}

\subsection{Flight Software Overview}

The SECCHI flight software runs within a real-time, multi-tasking operating system, VxWorks, that provides all of the software services needed by the instruments. This includes handling commands from the spacecraft, housekeeping and science telemetry to the spacecraft, heater control, guide telescope control, instrument control, image scheduling and image processing. The code is derived from code used in NASA's Small Explorer (SMEX) series of satellites and from the SOHO/LASCO experiment which was then modified for the SECCHI instrument.

The VxWorks operating system provides the infrastructure for running multiple tasks and each of the major functions listed above are run as separate tasks that can be started, stopped or reloaded independently. This made modifying the heritage code easy since the code is naturally modular. VxWorks also provides inter-task communications, which have been developed into a software bus library, shared by all tasks. The heritage software provided a large amount of useful code which reduced our task to modifying the telemetry and command tasks for SECCHI hardware and STEREO spacecraft protocols, developing new tables, telemetry and commands within the SMEX framework and developing new SEC$\mathrm{CHI}$ code for image scheduling, image processing and control of the SECCHI hardware, specifically cameras, mechanisms, heaters, power, housekeeping board. The SECCHI flight software contains about 250,000 lines of code.

\subsection{Instrument Scheduling}

The STEREO spacecraft will use the NASA Deep Space Missions System for commanding and telemetry downlink. Only one or two 4-hour contacts per day per spacecraft are planned. During the contact time the uplink rate is $2 \mathrm{kbits} / \mathrm{s}$ shared between all instruments and the spacecraft, which means limited daily commanding and a need for handling large blocks of schedule. The distance of the spacecraft from Earth and the resulting light travel time curtails interactive operations so that the instrument must perform most operations by programmed sequences on-board the instrument. 
The SECCHI concept of operations leads to the following characteristics for image scheduling:

(1) Provide for an autonomous operation for long periods of time (1 week or more)

(2) Synchronize image taking between spacecraft in which every image is scheduled

(3) Handle some non-image taking operations in timeline (doors and script tasks)

(4) Manage timeline of image taking and events to 1 second resolution

(5) Accept relative time schedules e.g. take COR1 image at 1 hour, 1 minute and $10 \mathrm{sec}-$ onds from the current time

(6) Accept absolute time schedules e.g. take COR1 image at 12:01:10 A.M. Nov 15, 2007

(7) Compact the image schedule of commands to reduce the uplink load

(8) Use on-board tables to minimize the amount of information uplinked daily

(9) Provide blocks of schedule commands that may be reused and called from a daily schedule

(10) Provide the planned schedules as a simple text file with embedded comments to facilitate ground operations and communication via email with co-investigators.

The image scheduling software maintains a single timeline of observations for all five telescopes. The uplinked schedules are parsed and entered into the timeline either manually or automatically at midnight each day from a RAM disk file. Normally the automatic load is used. If the expected schedule is not found then a default schedule is loaded.

A normal image acquisition sequence consists of the following steps: set up the instrument mechanism configuration, clear the CCD, open the shutter for a specified time, read out the CCD and transmit the digitized signal to a buffer in the SEB, process the image for downlink. One CCD from the SCIP and one CCD from the HI can be read out at the same time. With a readout time of about 4 seconds, this is not a severe restriction. Exposures may overlap on all of the telescopes.

Schedules are designed on the ground using a software planning tool that uses the flight software tables as input. The planning tool is able to add, delete and insert images into its timeline, manage on-board resources such as RAM buffer space, downlink telemetry, computer usage, spacecraft solid state recorder (SSR) usage, times of image exposure and image readout. The tool can merge blocks of schedule to form daily schedules. One common use is to merge a special observing program with a synoptic schedule. The planning tool also schedules door operations in anticipation of spacecraft thruster firings and script tasks used for guide telescope calibration and mechanism calibration.

\subsection{Image Processing}

Once an image is taken, the image is moved from the camera buffer memory to the computer for image processing. The image processing task is a background task that processes one image at a time and produces an image file ready to downlink. Since there are 27 camera buffers it is possible for image taking to take data faster than it can be processed, so the task maintains a queue of images to be processed in the order they are taken.

There are 120 image processing functions including three major types of image compression, event detection and reporting, two types of cosmic ray scrubbing, automatic exposure control, automatic compression control, occulter and region of interest masks, adding and subtracting images and the ability to send images down any of four channels (real-time, space weather, solid state recorder 1 , solid state recorder 2). When the image is scheduled, image processing is selected by specifying a row from an image processing table where each row is a list of functions to be done in sequential order. There are 100 rows in the image processing table. New image processing tables can be uploaded in flight as needed. 
Image compression is the most important feature of the image processing. Four types are provided including no compression. The Rice method is a lossless compression providing about a factor of 2.2 times compression. $\mathrm{H}$-Compress is a lossy wavelet image compression with a variable image compression. ICER is a lossy wavelet image compression, which has the advantage of being able to specify the desired output size. ICER is predicted to produce useful images with a compression factor of up to 20. Rice and H-compress were used to compress the LASCO and EIT images and ICER was used on the Mars Exploration Rover (MER) mission.

In order to achieve the desired signal to noise ratio, the $\mathrm{HI}$ images require longer exposure times (about 30 minutes) than the SCIP images. To eliminate the dark current the long exposures are obtained by exposing the CCDs for short times (up to 1 minute) and then summing the desired number of images in the on-board computer. The summing involves the following steps: remove the electronic offset, scrub the images of cosmic ray impacts, perform a $2 \times 2$ pixel sum to create a $1024 \times 1024$ image and then add the binned image to the image sum. The cosmic ray impacts (about 45 pixels/s) must be removed prior to summing. Two types of cosmic ray scrubbing are provided. The first does a two-image compare of each pixel of the current image to the previous image. If the current pixel is within the photon noise range of the last pixel it is accepted otherwise it is replaced with the older pixel. The second method does a three-image compare and chooses the median value. The images are then summed and added to $1024 \times 1024 \times 32$ bit image buffers. Successive HI images, typically tens of images, are added to the image buffer until the 32 bit image buffer is sent down using Rice image compression. The cosmic ray scrubbing and image summing for $\mathrm{HI}$ is the single most demanding task for the on-board computer.

After image processing is done, the completed files are put on a $52 \mathrm{MB}$ RAM disk for the telemetry task to send the file to the spacecraft over the MIL-STD 1553 spacecraft bus. The bus transfers roughly $1 \mathrm{MB}$ per minute to the spacecraft. A full sized SECCHI uncompressed image is $8 \mathrm{MB}$ in size. Using an image compression factor of $20 \times$ means that a full sized image will downlink to the spacecraft in just 24 seconds and that the RAM buffer can hold about 120 images.

\section{SECCHI Concept of Operations}

\subsection{Overview}

The SECCHI Payload Operations Center (POC) will be located at NRL for all normal operations, except for critical instrument debugging when it will be located at the Johns Hopkins University Applied Physics Laboratory (APL). The POC sends commands to and receives telemetry from the instrument via the Mission Operations Center (MOC), located at APL. SECCHI uses the Integrated Test and Operations System (ITOS) software hosted on Sun Blade workstations running Solaris (one for each spacecraft) for commanding and telemetry processing. Commands to be transmitted to the instrument are sent to the MOC. The MOC performs simple verification tests and then queues the commands for transmission to the spacecraft via the Deep Space Mission System (DSMS). Finally, the Command and Data Handling $(\mathrm{C} \& \mathrm{DH})$ process on the spacecraft forwards the commands to SECCHI over the 1553 bus. During normal operations, commands will be sent to the MOC at least 8 hours in advance of a daily track that lasts from 3.5 to 5 hours, depending on the phase of the mission. The daily tracks are nominally centered every 24 hours for each observatory.

Most housekeeping and science data are recorded on the onboard solid-state recorder (SSR) for later transmission to the ground during the daily track. The nominal SECCHI 
real-time data rate is 3.6 kilobits per second (kbps), and will be used predominantly for critical housekeeping data. Real-time telemetry is forwarded immediately to the MOC, and is made available to the POC via a TCP/IP connection. All real-time and SSR playback telemetry is forwarded from the DSMS to the MOC after completion of each track. The MOC maintains an archive of all instrument telemetry for 30 days. The SECCHI POC will retrieve the telemetry daily, after the MOC has produced cleaned and merged Level-0 files. The MOC will split the daily telemetry into 6 files, each containing four hours of SECCHI telemetry. Because data for a given day may require more than one track to be completely transmitted to the ground, the Level-0 files are regenerated each day for the two preceding days. The final Level-0 files are generated by the MOC after 30 days. The SECCHI POC will download those files also. The pipeline processing will be applied daily to the quick-look Level-0 files and again after the final Level-0 files are prepared.

\subsection{SECCHI Data Allocation and SSR utilization}

Table 9 shows the peak data rate at which the C\&DH collects telemetry data from SECCHI over the 1553 bus, and the SSR volume and downlink bandwidth allocated to SECCHI at various stages during the nominal two-year mission. Note that the allocations in this table are based on the average data volume guaranteed by the MOC to be downlinked during each daily track. The actual daily data volumes achieved during the mission may be higher than the nominal SSR allocations. The SECCHI SSR allocation of $6553 \mathrm{Mbits}$ includes margin to account for cases where the daily tracks are separated by more than the nominal 24 hours, and is sufficient to hold up to 36 hours of data at an average SSR write rate of $50 \mathrm{kbps}$.

The SSR allocation is divided into two partitions for science data and one for space weather data. The SSR1 partition is nominally $80 \%$ of the total allocation for science data. When SSR1 partition is full, no further data may be written to it until all data on the partition has been transmitted to the ground. The SSR 2 partition is nominally $20 \%$ of the total allocation for science data. The SSR2 partition functions like a circular buffer, and will overwrite the oldest data when full. The flight SW controls which packets get sent to the SSR2 and can stop writing if it detects an event trigger for example.

It is anticipated that SSR1 will be used for a synoptic program that will maintain synchronized observations on both spacecraft, and that will remain unchanged for extended periods of time throughout the mission. A sample synoptic program that fits within the nominal SSR1 allocation in the first 14 months on heliocentric orbit is shown in Table 10. The program shown in Table 10 is only for illustration of the type of observations that might be taken. The exact program will be decided after launch and the commissioning period and can change throughout the mission. SSR2 will be used for special observing programs, for

Table 9 SECCHI data rates and SSR allocations

\begin{tabular}{lll} 
Table 9 SECCHI data rates and & & \\
\cline { 2 - 2 } SSR allocations & 1553 bus TM peak data rate & $152 \mathrm{kbps}$ \\
& SSR partitions & \\
& SSR1 & 5162 Mbits \\
SSR2 & 1291 Mbits \\
& Downlink data volume & \\
Mission time ${ }^{1}$ & Average daily data rate \\
& Months 0-14 & 54 kbps \\
${ }^{1}$ Months after entering & Months 14-18 & $50 \mathrm{kbps}$ \\
heliocentric orbit & Months 18-24 & $45 \mathrm{kbps}$ \\
\hline
\end{tabular}

${ }^{1}$ Months after entering heliocentric orbit 
Table 10 Sample daily synoptic program for the first 14 months on heliocentric orbit

\begin{tabular}{llcllc}
\hline Telescope & $\begin{array}{l}\text { \# Images and } \\
\text { size (pixels) }\end{array}$ & $\begin{array}{l}\text { Cadence } \\
(\text { minutes })\end{array}$ & $\begin{array}{l}\text { Total } \\
\text { images/day }\end{array}$ & $\begin{array}{l}\text { Compression } \\
\text { factor }\end{array}$ & Total Mbits/day \\
\hline EUVI & $22 \mathrm{k} \times 2 \mathrm{k}$ & 4 & 576 & 40.0 & 845.6 \\
& $42 \mathrm{k} \times 2 \mathrm{k}$ & 20 & 288 & 10.0 & 1691.1 \\
COR1 & $31 \mathrm{k} \times 1 \mathrm{k}$ & 8 & 540 & 10.0 & 792.7 \\
COR2 & $31 \mathrm{k} \times 1 \mathrm{k}$ & 15 & 216 & 10.0 & 317.1 \\
& $32 \mathrm{k} \times 2 \mathrm{k}$ & 60 & 72 & 10.0 & 422.8 \\
HI1 & $11 \mathrm{k} \times 1 \mathrm{k}$ & 60 & 24 & 2.5 & 211.4 \\
HI2 & $11 \mathrm{k} \times 1 \mathrm{k}$ & 120 & 12 & 2.5 & 105.7 \\
Total & & & 1728 & & 4386.4 \\
\hline
\end{tabular}

example those that require high-cadence observations with one or more telescopes for a short period of time. These may or may not be duplicated on the other spacecraft. For example, if the trigger to stop recording is based on an on-board CME detection, there is no guarantee that both spacecraft will see the same event at the same time.

\subsection{Observation Planning}

The SECCHI flight software has enabled a very flexible program and supports the acquisition of over 5000 images each day for each spacecraft. The relative cadence of the various image types is the main variation that affects the planning. The basic observing philosophy for SECCHI consists of two observing programs that run simultaneously on both observatories. The first ("synoptic") program schedules every observation by time, is identical on both observatories, and occupies about $80 \%$ of the available telemetry. The second ("campaign") program is more flexible, allowing higher data rates for limited periods of time. It writes to SSR2, the overwriting telemetry buffer. Simultaneous observations from both observatories are still desirable in this mode, but are not a requirement. The lower cadence data from the synoptic program from both observatories remains undisturbed.

It is important to keep in mind that the scientific emphasis of the STEREO mission is constantly changing as the two observatories drift apart. Since observations at a specific spacecraft separation angle can't be repeated, observations must be carefully planned in advance. We will favor the EUVI observations early in the mission, when the separation angle between the observatories is small enough for stereoscopic imaging in the classical sense, and when the STEREO telemetry rate is highest due to the proximity to Earth. During that time, the EUVI will focus on CME initiation studies using high cadence imaging.

Planning for SECCHI observations will occur in multiple stages in coordination with other STEREO instruments and the MOC, with more detailed plans established at each stage. Broad science plans and priorities will be established at quarterly meetings of the STEREO Science Working Group (SWG) and at the SECCHI team meetings. The plans will be posted on the web and will be refined during monthly meetings of the Science Operations Working Group (SOWG), after which a schedule of observations will be generated. The detailed plans will be finalized at weekly teleconferences, with participation from the spacecraft, all instrument teams and the SSC. The SECCHI observing plans for one day up 
to a week will be generated using the SECCHI Planning Tool. This tool is a set of Interactive Data Language (IDL) procedures that provides a graphical user interface to allow the operator to generate a file of schedule commands for both spacecraft to be sent to the MOC via the ITOS software. The planning tool performs constraint checking (for example, a warning is generated if simultaneous readouts from two CCDs controlled from the same CEB are scheduled), and monitors both internal buffer space and telemetry usage for each telescope. It is based on a similar tool that has been used in planning LASCO and EIT observations on the SOHO mission for over 10 years. The planned schedule can be displayed on the Web both in graphical format and as a text file.

\subsection{Beacon Mode}

SECCHI space weather data will be broadcast continuously at a rate of 504 bps over the beacon channel. These data will consist of a subset of images from each telescope. In order to fit within the low bandwidth, the images need to be highly compressed, probably binned to a low spatial resolution, as well as a subset of event messages that can be used to monitor instrument status outside of the regular track periods. The exact compression techniques will be determined after launch. The SSC is responsible for collecting these data from the various ground stations that receive them, processing the data and serving them on the Web.

\subsection{SECCHI Campaigns}

For two or more periods totaling four weeks during the mission, there will be an additional track, beginning 12 hours after the start of the normal daily track. The effect of this additional track is to double the amount of telemetry that SECCHI is able to downlink each day. These 'SECCHI Campaigns' will enable high-cadence observations targeted to specific science objectives.

\subsection{Routine Processing and Data Products}

Routine processing of SECCHI data will take place at the POC (for real-time and socket playback data) and at the SECCHI Data Processing Facility (DPF, for Level-0 packet files) at NRL. Science data will be processed into FITS files, consisting of a binary data array preceded by a header, with one file per image. No calibrations will be applied to the data during routine processing, and the resulting FITS file will be referred to as Level-0.5. FITS files generated from real-time data are referred to as 'quick-look'. FITS files generated from socket playback or Level-0 files will be referred to as 'preliminary'. When the final Level-0 files are available from the MOC after 30 days, the resulting FITS files will be referred to as 'final'. All final Level-0.5 FITS files will be archived at NRL, and will also be delivered electronically to the SSC and to Co-Investigator institutions. Processing of data to Level-1 and higher levels will be done by individual users, using IDL routines and auxiliary data that will be freely distributed as part of the SolarSoft software library and database. Some higher level data products, such as polarized brightness images from COR1 and COR2, and Carrington maps, will be generated routinely by the DPF. Additional data products, including browse images, movies, lists of events such as CMEs and comets, housekeeping tables and long-term trending plots of key instrument performance parameters will also be generated routinely and made available electronically. 


\subsection{SECCHI Data Policy}

The SECCHI data policy is to have completely open access to all data, including the calibration data and procedures necessary to calibrate and process the data to higher levels. SECCHI images will be available as soon as the routine processing steps have been completed. This is estimated to be within 30 minutes of receipt of all data necessary to form an image. The image data will be accessible via the Web using a searchable database. It will be possible to generate queries to select and download data of interest using FITS header keywords as parameters. Temperatures, voltages, and other housekeeping parameters will also be accessible through database queries.

Acknowledgements We gratefully acknowledge the hard work and dedication of the literally hundreds of people who contributed to the SECCHI program. The SECCHI instrument was constructed by a consortium of institutions: the Naval Research Laboratory (NRL, Washington, DC, USA), the Lockheed Martin Solar and Astrophysical Laboratory (LMSAL, Palo Alto, CA, USA), the NASA Goddard Space Flight Center (GSFC, Greenbelt, MD, USA), the Max Planck Institüt für Sonnensystemforschung (MPS, Lindau, Germany), the Centre Spatial de Liege (CSL, Angleur, Belgium), the University of Birmingham (UB, Birmingham, UK), the Rutherford Appleton Laboratory (RAL, Didcot, UK), the Institut d'Optique (IOTA, Orsay, France), the Institute d'Astrophysique Spatiale (IAS, Orsay, France).

NRL is the lead institution in the consortium and was responsible for the supplying the COR2 coronagraph, the experiment electronics, the CCDs and focal plane assemblies, the flight and ground software, the integration and testing of the instrument and the overall program management. LMSAL was responsible for providing the EUVI telescope, the GT, the mechanism electronics box and mechanisms for the COR1, COR2 and EUVI shutters and wheels. The GSFC was responsible for providing the COR1 telescope.

The USA effort was supported by the NASA Solar Terrestrial Physics STEREO Program under DPR S-13631-Y. The program managers at GSFC were Abby Harper, Haydee Moldanado and for the last 3 years of the program, Nicholas Chrissotimos. The program manager for instruments was Michael Delmont and the SECCHI instrument manager was Bernie Klein. We are indebted to their efforts and to those of the other members of the STEREO project. The NRL effort was also supported by the USAF Space Test Program and the Office of Naval Research.

At NRL, the COR2 subsystem design, fabrication and testing was done by Dennis Socker, Simon Plunkett, Angelos Vourlidas, Clarence Korendyke, Edward Shepler, Jeff Morrill of NRL, Qian Gong, Robert Hagood, Paul Nikulla and Pete Patterson of Swales, Inc, Martin Koomen of Sachs-Freeman, Robert Moye, David Roberts, Arnaud Thernisien and Robin Colaninno of Artec and Marc Westcott of Tropel. The FPA was designed and tested by Benjamin Au of Praxis, Dan Moses, Jeffrey Newmark of NRL, and Paul Nikulla and Miles Newman of Swales. The FPAs were fabricated at the University of Birmingham by Chris Eyles, Clive Longstaff, and John Bryant. The CCDs were procured from e2V and were calibrated by Richard Kroeger of NRL. The SEB hardware was designed and tested by Sean Lynch, Robert Skalitzky and Amy Hurley of NRL, Drew Roberts, Greg Clifford, and Dennis Silver of Silver Engineering, Inc., and Dale Bankus of OSC. The SEB flight software was designed and developed by Dennis Wang of Interferometrics, Inc., and Ladd Wheeler and Joel Chiralo of the Hammers Company and Matthew Hayden of NRL. The harness was designed and built by Susie LaCava, James (Chris) Garner, and Richard Cooksey of NRL and Ralph Hopkins of Honeywell. The SCIP door design and testing was supported by Stephen Koss and Paul Oppenheimer of NRL. The SECCHI environmental testing was performed by Michael VanHerpe, Robert Haynes and Michael Obara of NRL. Contamination control support was provided by Therese Errigo, Radford Perry and Andrew Uhl of Swales Inc. Configuration management was provided by John Tota, Nick Virmani, Ken Slocum, Stu Sweitzer of Swales. SECCHI project management was provided by Rebecca Baugh, Donald McMullin and Dale Harris of Praxis, Inc. System engineering was provided by Timothy Carter and Benjamin Au of Praxis and James Garner of NRL. The SCIP thermal design was performed by Miles Newman of Swales.

At LMSAL, the EUVI and GT telescopes were designed, fabricated, and tested by James Lemen, JeanPierre Wuelser, Jacob Wolfson, Glenn Gradwohl, Theodore Tarbell, Joseph Cannon, Noah Katz, Dunagan Pearson. The mechanisms were designed and built by Augustus Moore and David Akin. The MEB and GT electronics were designed by Larry Springer, George Rossi and Russell Lindgren. Contamination support was provided by Syndie Meyers and thermal support by Rose Deguzman. Program management was done by Dexter Duncan, Larry Springer and Frank Friedlaender. Configuration management and system engineering support was performed by Brock Carpenter and Claude Kam.

At GSFC, the COR1 was designed, fabricated and tested by Joseph Davila, Eric Mentzell, Kim Mehalik, Julie Crooke, Rebecca Derro, Chris St Cyr, William Thompson of GSFC and Michael Hersh and Miles 
Newman of Swales. Program management was performed by Lawrence Orwig and system engineering by Richard Wiesenberg.

The German science and hardware contributions to SECCHI-the project STEREO/Corona-is supported by the German "Bundesministerium für Bildung und Forschung" through the "Deutsche Zentrum für Luft- und Raumfahrt e.V.” (DLR, German Space Agency). The Max-Planck-Institute für Sonnensystemforschung supplied the aperture doors for the SCIP telescopes.

The UK contribution to STEREO was funded by the Particle Physics and Astronomy Research Council (PPARC) and we also acknowledge significant support for the Heliosphere Imager from the School of Physics and Astronomy, University of Birmingham. The HI and CEB program management was led by Richard Harrison and James Lang at RAL. At the University of Birmingham, the HI design, fabrication and testing was performed by Christopher Eyles, CEB hardware was designed by Nick Waltham, James King, Andrew Marshall and Matthew Clapp. The CEB software was developed by Duncan Drummond and John Rainnie. The CEBs were fabricated and tested at the University of Birmingham by Chris Eyles, John Bryant and David Smith. The HI thermal design was performed by Helen Mapson-Menard initially and completed by Bryan Shaughnessy of RAL.

The French teams were supported by the Centre National d'Etudes Spatiales and the Centre National de la Recherche Scientifique. At IOTA, the design and fabrication of the EUVI multilayers was performed by Marie-Françoise Ravet, Françoise Bridou, Franck Delmotte and Arnaud Jerome and the polishing/figuring of the EUVI mirrors was performed by Jean-Yves Clotaire, Gilles Colas, Michel Lamare, Raymond Mercier and Michel Mullot. The testing and calibration of the multilayer mirror performance was performed at IAS by Jean-Pierre Delaboudiniere, Frederic Auchere, Philippe Bouyries and Xueyan Song.

The Belgian effort was supported by the Belgian Science Policy Office. The HI optical design, environmental testing and calibration (stray-light and photometric) was performed at Centre Spatial de Liege by Jean-Marc Defise, Jean-Philippe Halain, Emmanuel Mazy, Philippe Ronchain and Pierre Rochus.

\section{References}

D. Akin, R. Horber, C.J. Wolfson, Three high duty cycle, space-qualified mechanisms, in NASA Conf. Pub., vol. 3205 (1993)

G.E. Brueckner, R.A. Howard, M.J. Koomen, C.M. Korendyke, D.J. Michels, J.D. Moses, D.G. Socker, K.P. Dere, P.L. Lamy, A. Llebaria, M.V. Bout, R. Schwenn, G.M. Simnett, D.K. Bedford, C.J. Eyles, Solar Phys. 162, 357-402 (1995)

A. Buffington, B.V. Jackson, C.M. Korendyke, Appl. Opt. 35, 6669-6673 (1996)

K.P. Dere, E. Landi, H.E. Mason, B.C. Monsignori Fossi, P.R. Young, CHIANTI—an atomic database for emission lines. Astron. Astrophys. Suppl. Ser. 125, 149 (1997)

J.M. Defise, J.P. Halain, E. Mazy, P. Rochus, R.A. Howard, J.D. Moses, D.G. Socker, R.A. Harrison, G.M. Simnett, Design and tests for the heliospheric imager of the STEREO mission, in Innovative Telescopes and Instrumentation for Solar Astrophysics, ed. by S.L. Keil, S.V. Avakyan. Proc. SPIE, vol. 4853 (SPIE, Bellington, 2003), pp. 12-22

J.P. Delaboudinière et al., EIT: extreme-ultraviolet imaging telescope for the SOHO mission. Sol. Phys. 162, 291-312 (1995)

C.J. Eyles, G.M. Simnett, M.P. Cooke, B.V. Jackson, A. Buffington, P.P. Hick, N.R. Waltham, J.M. King, P.A. Anderson, P.E. Holladay, Sol. Phys. 217, 319-347 (2003)

E.G. Gibson, The Quiet Sun, SP-303, National Aeronautics and Space Administration (1973)

B.N. Handy et al., The transition region and coronal explorer. Sol. Phys. 187, 229-260 (1999)

R.A. Harrison, C.J. Davis, C.J. Eyles, The STEREO heliospheric imager: how to detect CMEs in the heliosphere. Adv. Space Res. 36, 1512-1523 (2005)

R.A. Howard, Historical perspective on coronal mass ejections, in Solar Eruptions and Energetic Particles, ed. by N. Gopalswamy. Geophysical Monograph Series, vol. 165 (American Geophysical Union, Washington, 2006), pp. 7-13

R.A. Howard, J.D. Moses, D.G. Socker, the SECCHI consortium, Sun-Earth connection coronal and heliospheric investigation (SECCHI), in Instrumentation for UV/EUV Astronomy and Solar Missions, vol. 4139, ed. by S. Fineschi, C.M. Korendyke, O.H. Siegmund, B.E. Woodgate (SPIE, Bellingham, 2000), pp. 259-283

B. Lyot, The study of the solar corona and prominences without eclipses (George Darwin Lecture, 1939). Mon. Not. R. Astron. Soc. 99, 580-594 (1939)

J.S. Morrill et al., Calibration of the SOHO/LASCO C3 white light coronagraph. Sol. Phys. 233, 331-372 (2006) 
E. Pitz, C. Leinert, H. Link, N. Salm, HELIOS zodiacal light experiment, in Interplanetary Dust and Zodiacal Light. IAU Colloq., vol. 31 (Cambridge University Press, Cambridge, 1976), pp. 19-23

M.F. Ravet, F. Bridou, X. Zhang-Song, A. Jerome, F. Delmotte, M. Bougnet, J.P. Delaboudinière, Ion beam deposited Mo/Si multilayers for EUV imaging applications in astrophysics. Proc. SPIE 5250 (2003)

K. Saito, A.I. Poland, R.H. Munro, A study of the background corona near solar minimum. Sol. Phys. 55, 121-134 (1977)

D.G. Socker, R.A. Howard, C.M. Korendyke, G.M. Simnett, D.F. Webb, The NASA solar terrestrial relations observatory (STEREO) mission heliospheric imager, in Instrumentation for UV/EUV Astronomy and Solar Missions, vol. 4139, ed. by S. Fineschi, C.M. Korendyke, O.H. Siegmund, B.E. Woodgate (SPIE, Bellingham, 2000), pp. 284-293

R.A. Stern, the SXI Team, Solar X-ray imager for GOES. Proc. SPIE 5171 (2003)

K. Strong, T. Bruner, M. Tarbell, A. Title, C. Wolfson, TRACE-the transition region and coronal explorer. Space Sci. Rev. 70, 119 (1994)

S.J. Tappin, A. Buffington, M.P. Cooke, C.J. Eyles, P.P. Hick, P.E. Holladay, B.V. Jackson, J.C. Johnston, T. Kuchar, D. Mizuno, J.B. Mozer, S. Price, R.R. Radick, G.M. Simnett, D. Sinclair, N.R. Waltham, D.F. Webb, Geophys. Res. Lett. 31, L02802 (2003). doi:10.1029/2003GL018766

W.T. Thompson, J.M. Davila, R.R. Fisher, L.E. Orwig, J.E. Mentzell, S.E. Hetherington, R.J. Derro, R.E. Federline, D.C. Clark, P.T.C. Chen, J.L. Tveekrem, A.J. Martino, J. Novello, R.P. Wesenberg, O.C. St Cyr, N.L. Reginald, R.A. Howard, K.I. Mehalick, M.J. Hersh, M.D. Newman, D.L. Thomas, G.L. Card, D.F. Elmore, COR1 inner coronagraph for STEREO-SECCHI, in Innovative Telescopes and Instrumentation for Solar Astrophysics, ed. by S.L. Keil, S.V. Avakyan. Proc. SPIE, vol. 4853 (SPIE, Bellingham, 2003), pp. 1-11

D.L. Windt, R.C. Catura, Multilayer characterization at LPARL. Proc. SPIE 984, 132-139 (1988)

J.-P. Wülser et al., EUVI: the STEREO-SECCHI extreme ultraviolet imager. Proc. SPIE 5171 (2003)

P.R. Young, G. Del Zanna, E. Landi, K.P. Dere, H.E. Mason, M. Landini, CHIANTI-an atomic database for emission lines. Astrophys. J. Suppl. Ser. 144, 135-152 (2003) 